\title{
Attenuation of Sound
}

\section{Contents}

14.1 An Almost Correct Expression for Viscous Attenuation ................ 674

14.2 Bulk Thermoviscous Attenuation in Fluids ...................... 677

14.3 Classical Thermoviscous Attenuation $\ldots \ldots \ldots \ldots \ldots \ldots \ldots \ldots \ldots \ldots \ldots, 681$

14.4 The Time-Dependent Equation of State $\ldots \ldots \ldots \ldots \ldots \ldots \ldots \ldots \ldots \ldots \ldots$

14.5 Attenuation due to Internal Relaxation Times ..................... 684

14.5.1 Relaxation Attenuation in Gases and Gas Mixtures ...................... 687

14.5.2 Relaxation Attenuation in Fresh and Salt Water ...................... 688

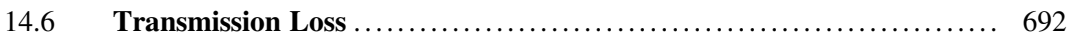

14.6.1 Short and Very Short Wavelengths ............................. 692

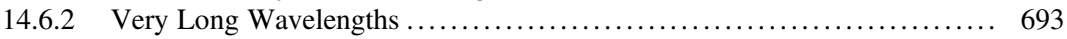

14.7 Quantum Mechanical Manifestations in Classical Mechanics ........... 693

References ..................................................... 697

There are four mechanisms that cause sound energy to be absorbed and sound waves to be attenuated as they propagate in a single-component, homogeneous fluid:

- Viscous (shear) effects in bulk fluids

- Thermal conduction in bulk fluids

- Molecular absorption in bulk fluids

- Thermoviscous boundary layer losses

The decrease in the amplitude of acoustical disturbances or in the amplitude of vibrational motion (due to dissipative mechanisms) has been a topic of interest throughout this textbook. In this chapter, we will capitalize on our investment in such analyses to develop an understanding of the attenuation of sound waves in fluids that are not influenced by proximity to solid surfaces. Such dissipation mechanisms are particularly important at very high frequencies and short distances or very low frequencies over geological distances.

The parallel addition of a mechanical resistance element to the stiffness and mass of a simple harmonic oscillator led to an exponential decay in the amplitude of vibration with time in Sect. 2.4. The 
(mechanically) series combination of a stiffness element and a mechanical resistance in the Maxwell model of Sect. 4.4.1, and in the Standard Linear Model of viscoelasticity in Sect. 4.4.2 introduced the concept of a relaxation time, $\tau_{R}$, that had significant effects on the elastic (in-phase) and dissipative (quadrature) responses as a function of the nondimensional frequency, $\omega \tau_{R}$. Those response curves were "universal" in the sense that causality linked the elastic and dissipative responses through the Kramers-Kronig relations, as presented in Sect. 4.4.4.

That relaxation time perspective, along with its associated mathematical consequences, will be essential to the development of expressions for attenuation of sound in media that can be characterized by one or more relaxation times related to those internal degrees of freedom that make the equation of state a function of frequency. Examples of these relaxation time effects include the rate of collisions between different molecular species in a gas (e.g., nitrogen and water vapor in air), the pressure dependence of ionic association-dissociation of dissolved salts in seawater (e.g., $\mathrm{MgSO}_{4}$ and $\mathrm{H}_{3} \mathrm{BO}_{3}$ ), and evaporation-condensation effects when a fluid is oscillating about equilibrium with its vapor (e.g., fog droplets in air or gas bubbles in liquids).

The viscous drag on a fluid oscillating within the neck of a Helmholtz resonator, combined with the thermal relaxation of adiabatic temperature changes at the (isothermal) surface of that resonator's compliance, led to energy dissipation in lumped-element fluidic oscillators in Sect. 9.4.4, producing damping that limited the quality factor of those resonances in exactly the same way as mechanical resistance limited the quality factor of a driven simple harmonic oscillator, which was first introduced as a consequence of similitude (i.e., dimensional analysis) in Sect. 1.7.1.

The thermoviscous boundary layer dissipation, summarized in Eq. (9.38), was used to calculate the attenuation of plane waves traveling in a waveguide in Sect. 13.5.5. As will be demonstrated explicitly in this chapter, thermoviscous boundary layer losses provide the dominant dissipation mechanism at low frequencies (i.e., lumped element systems and waveguides below cut-off) for most laboratorysized objects (including the laboratory itself when treated as a three-dimensional enclosure). For fluid systems that are not dominated by dissipation on solid surfaces in close proximity to the fluids they contain, the dissipation due to losses within the fluid itself (i.e., bulk losses) can be calculated directly from the hydrodynamic equations of Sect. 7.3.

To reintroduce the concepts complex wavenumber or complex frequency that typically characterize the attenuation of sound over space or time, a simple solution of the Navier-Stokes equation will first be derived. That approach will not provide the correct results for attenuation of sound, even in the absence of relaxation effects, because it does not properly take into account the relationship between shear deformation and hydrostatic compression in fluids that are necessary to produce plane waves (see Fig. 14.1). That relationship was used to relate the modulus of unilateral compression for isotropic solids (aka the dilatational modulus) to other isotropic moduli in Sect. 4.2.2 and in Fig. 4.3. The complete solution for bulk losses due to a fluid's shear viscosity, $\mu$; thermal conductivity, $\kappa$; and the relaxation of internal degrees of freedom (i.e., "bulk viscosity), $\zeta$, will follow that nearly correct introductory treatment and will be based upon arguments related to entropy production, like the analysis in Sect. 9.3.3.

\subsection{An Almost Correct Expression for Viscous Attenuation}

Because we started with a complete hydrodynamic description of homogeneous, isotropic, singlecomponent fluids using the Navier-Stokes equation, we are now well-prepared to investigate the dissipation mechanisms that attenuate the amplitude of sound waves propagating far from the influence of any solid boundaries. A one-dimensional linearized version of the Navier-Stokes equation (9.2) is reproduced below: 


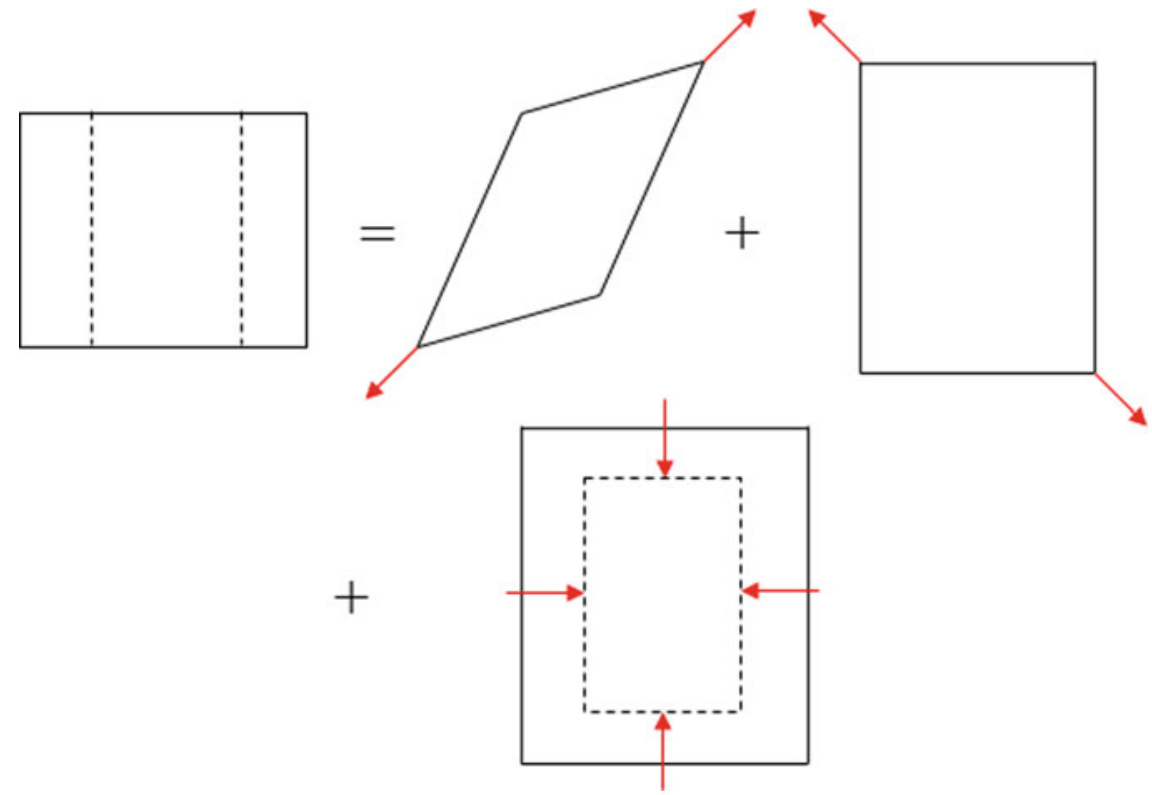

Fig. 14.1 Schematic two-dimensional representation of the combination of shear and hydrostatic deformations necessary to produce the unilateral compression of a fluid element, corresponding to a plane wave, shown at the upper left. If the original fluid parcel at the upper left is a square, shown by the solid lines, then the compression accompanying a plane wave does not change the upper and lower boundaries, but would require that the two vertical boundary lines contract to the positions indicated by the two dashed lines. This deformation can be accomplished by first shearing the square fluid element along one diagonal and then shearing it again along the other diagonal, as indicated by the arrows. Those two deformations result in making the square into a rectangle. When the rectangle is subjected to a hydrostatic compression, that rectangle is compressed into the required shape

$$
\rho_{m} \frac{\partial v_{1}(x, t)}{\partial t}+\frac{\partial p_{1}(x, t)}{\partial x}-\mu \frac{\partial^{2} v_{1}(x, t)}{\partial x^{2}}=0
$$

The linearized, one-dimensional continuity equation (10.1) is not affected by the inclusion of viscosity in the Navier-Stokes equation.

$$
\frac{\partial \rho_{1}(x, t)}{\partial t}+\rho_{m} \frac{\partial v_{1}(x, t)}{\partial x}=0
$$

Since the thermal conductivity of the fluid is ignored, $\kappa=0$, the linearized version of the adiabatic equation of state can still be invoked to eliminate $\rho_{1}(x, t)$ in favor of $p_{1}(x, t)$, allowing expression of the continuity equation in terms of the same variables used in Eq. (14.1).

$$
\begin{aligned}
& \rho_{1}(x, t)=\left(\frac{\partial \rho}{\partial p}\right)_{s} p_{1}(x, t)=\frac{p_{1}(x, t)}{c^{2}} \\
& \Rightarrow \quad \frac{1}{\rho_{m} c^{2}} \frac{\partial p_{1}(x, t)}{\partial t}+\frac{\partial v_{1}(x, t)}{\partial x}=0
\end{aligned}
$$

As done so many times before, the dispersion relation, $\omega(k)$, will be calculated by assuming a rightgoing traveling wave to convert the homogeneous partial differential equations (14.1) and (14.3) to coupled algebraic equations. 


$$
\begin{aligned}
-j k p_{1}+\left(j \omega \rho_{m}+k^{2} \mu\right) v_{1} & =0 \\
j \omega \frac{p_{1}}{\rho_{m} c^{2}}-j k v_{1} & =0
\end{aligned}
$$

There are now both real and imaginary terms in the coupled algebraic equations, unlike their nondissipative equivalents, such as Eqs. (10.16) and (10.17). The existence of a nontrivial solution to Eq. (14.4) requires that the determinant of the coefficients vanish.

$$
\left|\begin{array}{cc}
\frac{j \omega}{\rho_{m} c^{2}} & -j k \\
-j k & \left(j \omega \rho_{m}+k^{2} \mu\right)
\end{array}\right|=0
$$

The evaluation of this determinant leads to the secular equation that will specify the complex wavenumber, $\boldsymbol{k}$, in terms of the angular frequency, ${ }^{1} \omega$.

$$
\left(\frac{\omega}{c}\right)^{2}=k^{2}\left(1+\frac{j \omega \mu}{\rho_{m} c^{2}}\right)
$$

If $\omega \mu / \rho_{m} c^{2}<<1$, the binomial expansion can be used twice to approximate the spatial attenuation coefficient, $\alpha$.

$$
\mathbf{k} \cong \frac{\omega}{c}-j \frac{\omega^{2} \mu}{2 \rho_{m} c^{3}}=k-j \alpha_{\text {almost }}
$$

To remind ourselves that this result is not completely correct, this spatial attenuation coefficient has been designated $\alpha_{\text {almost }}=\omega^{2}\left(\mu / 2 \rho_{m} c^{3}\right)$. The form of this result, specifically the fact that the attenuation is proportional to $\omega^{2} / \rho_{m}$, suggests that experimental results could be plotted as a function of the square of frequency divided by mean pressure, as shown in Fig. 14.4.

It is also useful to notice that $\mu / \rho_{m} c^{2}$ in Eq. (14.6) has the units of time, so the "small parameter" in those binominal expansions of Eq. (14.6) is of the form $j \omega \tau_{\bar{\ell}}$. Equally important is the recognition that such a relaxation time, $\tau_{\bar{\ell}}$, is on the order of the collision time in a gas, based on the mean free path, $\bar{\ell}$, derived from simple kinetic theory of gases in Sect. 9.5.1 [1].

$$
\tau_{\bar{\ell}}=\frac{\bar{\ell}}{c} \cong \frac{3 \mu_{\text {gas }}}{\rho_{m} c^{2}}=\frac{3}{\gamma} \frac{\mu_{\text {gas }}}{p_{m}}
$$

This is different from the relaxation times, $\tau_{R}$, which can characterize the time dependence of the equation of state or the response of a viscoelastic medium described in Sect. 4 .4, where $\omega \tau_{R} \geqq 1$. At frequencies above $\omega_{\bar{\ell}} \cong\left(\tau_{\bar{\ell}}\right)^{-1}$, the assumptions that underlie the hydrodynamic approach are no longer valid (see Chap. 7, Problem 1). For air near room temperature and at atmospheric pressure, $\tau_{\bar{\ell}} \cong$ $400 \mathrm{ps}$, so $f=\omega / 2 \pi \cong 400 \mathrm{MHz}$. This is identical with the result obtained in Eq. (9.24) for the critical frequency, $\omega_{\text {crit }}$, at which sound propagation in air transitions from adiabatic at low frequencies to isothermal at high frequencies. The regime where $\omega \tau_{\bar{\ell}}>1$ becomes questionable within the context of a (phenomenological) hydrodynamic theory [1].

\footnotetext{
${ }^{1}$ The decision to treat $\omega$ as a real number, thus forcing the wavenumber, $\boldsymbol{k}$, to become a convenience (complex) number, is arbitrary. It leads to a spatial attenuation coefficient that is related to the imaginary component of the wavenumber. Treating the wavelength, $\lambda=2 \pi / k$, as a real number forces the frequency, $\omega$, to be a convenience number, thus producing a temporal attenuation coefficient. Of course, spatial-to-temporal conversions can be accomplished using the sound speed, as shown in Eq. (14.10).
} 
Unlike the complex wavenumbers of exponentially decaying thermal and viscous waves near boundaries, examined in Sects. 9.3.1 and 9.4.2, where $\mathfrak{R e}[\boldsymbol{k}]=? \mathrm{~m}[\boldsymbol{k}]$, most attenuation mechanisms in bulk fluids far from boundaries have $\mathfrak{R e}[\boldsymbol{k}] \gg ? \mathrm{~m}[\boldsymbol{k}]$ or $|\boldsymbol{k}| \gg \alpha$. If $\boldsymbol{k}$ is substituted into the expression for the pressure associated with a single-frequency one-dimensional plane wave traveling in the $+x$ direction, $p_{1}(x, t)$, it is easy to see that $\alpha$ leads to an exponential decay in the amplitude with propagation distance for the sound wave.

$$
p_{1}(x, t)=\mathfrak{R e}\left[\widehat{\mathbf{p}} e^{j(\omega t-\mathbf{k} x)}\right]=\mathfrak{R e}\left[\widehat{\mathbf{p}} e^{j[\omega t-(k-j \alpha) x]}\right]=e^{-\alpha x} \mathfrak{R e}\left[\widehat{\mathbf{p}} e^{j(\omega t-k x)}\right]
$$

Since space and time can be transformed by the sound speed, a real temporal attenuation coefficient can be defined, $\beta=\alpha c$, to describe the rate at which the amplitude of the plane wave decays in time.

$$
p_{1}(x, t)=e^{-\beta t} \mathfrak{R e}\left[\widehat{\mathbf{p}} e^{j(\omega t-k x)}\right] \quad \text { where } \beta=\alpha c
$$

Although the result for $\alpha_{\text {almost }}$ is not exactly correct, it does exhibit a feature of the correct result for the spatial attenuation coefficient that includes thermoviscous dissipation that is given by $\alpha_{\text {classical }}$ in Eq. (14.31) where internal relaxation effects are discussed in Sect. 14.5. ${ }^{2}$

Unlike the spatial attenuation coefficient for dissipation of plane waves in a waveguide, given in Eq. (13.78), which is proportional to $\sqrt{\omega}$, Eq. (14.7) shows that $\alpha_{\text {almost }}$ is proportional to the square of the frequency, as is $\alpha_{\text {classical }}$.

\subsection{Bulk Thermoviscous Attenuation in Fluids}

Although the previous results for $\alpha_{\text {almost }}$ are incomplete, it both has provided an introduction to the complex wavenumber, $\mathbf{k}$, that determines the attenuation distance and has introduced a relaxation time, $\tau_{\bar{\ell}}$, that sets an upper limit to the frequencies above which the continuum model of a fluid is not appropriate. One reason that previous result for the viscous attenuation is not complete is that we have ignored the fact that the fluid deformation corresponding to the passage of a plane wave requires the superposition of two shear deformations and a hydrostatic compression. This superposition of shear strain and hydrostatic strain is illustrated schematically in Fig. 14.1. Of course, the result for the attenuation coefficient, $\alpha_{\text {almost }}$, in Sect. 14.1, also does not yet include the thermal conductivity, $\kappa$, of the fluid.

To incorporate all of the dissipative effects in a fluid, it is necessary to start from the complete expression for entropy production in a single-component homogeneous fluid. The mechanical energy dissipation, $E_{\text {mech }}$, is the maximum amount of work that can be done in going from a given non-equilibrium state of energy, $E_{o}$, back to equilibrium, $E(S)$, which occurs when the transition is reversible (i.e., without a change in entropy) [2]. $\dot{E}_{\text {mech }}$ is the rate at which the mechanical energy is dissipated by the periodic transitions from the non-equilibrium state to the equilibrium state as orchestrated by the wave motion.

$$
\dot{\Pi}_{m e c h}=-\dot{E}(S)=-\left(\frac{\partial E}{\partial S}\right) \dot{S}=T_{m} \dot{S}
$$

The right-most expression in Eq. (14.11) uses the fact that the derivative of the energy with respect to the entropy is the equilibrium value of the mean absolute temperature, $T_{m}$. The entropy equation

\footnotetext{
${ }^{2}$ Since inert gases have no internal degrees of freedom, $\alpha_{\text {classical }}$ provides their entire attenuation constant.
} 
(7.43) can be written so that the shear stresses and the hydrostatic stresses can be expressed symmetrically in Cartesian components. ${ }^{3}$

$$
\rho T\left(\frac{\partial s}{\partial t}+\vec{v} \cdot \vec{\nabla} s\right)=\nabla \cdot(\kappa \vec{\nabla} T)+\frac{1}{2} \mu\left(\frac{\partial v_{i}}{\partial x_{k}}+\frac{\partial v_{k}}{\partial x_{i}}-\frac{2}{3} \frac{\partial v_{i}}{\partial x_{i}}\right)^{2}+\zeta(\nabla \cdot \vec{v})^{2}
$$

The two cross derivatives, $\left(\partial v_{i} / \partial x_{k}\right)$ and $\left(\partial v_{k} / \partial x_{i}\right)$, represent the two shear deformations with the hydrostatic component removed: $2 / 3\left(\partial v_{i} / \partial x_{i}\right)$ [3]. The square of the hydrostatic deformation is represented by $(\nabla \cdot \vec{v})^{2}$. The hydrostatic deformation is multiplied by a new positive scalar coefficient, $\zeta$, that must have the same units as the shear viscosity [Pa-s].

Having the form of a conservation equation (see Sect. 10.5), the right-hand side of Eq. (14.12) represents the rate of entropy production, $\dot{S}$, caused by thermal conduction, viscous shear, and some possible entropy production mechanism (unspecified at this point but eventually related to the time dependence of the equation of state) associated with the hydrostatic deformation. Using Eq. (14.12), the dissipated mechanical power, $\Pi_{\text {mech }}$, can be evaluated by integrating over a volume element that includes the plane wave disturbance, $d V$.

$$
\Pi_{\text {mech }}=-\frac{\kappa}{T_{m}} \int(\nabla T)^{2} d V-\frac{\mu}{2} \int\left(\frac{\partial v_{i}}{\partial x_{k}}+\frac{\partial v_{k}}{\partial x_{i}}-\frac{2}{3} \frac{\partial v_{i}}{\partial x_{i}}\right)^{2} d V-\zeta \int(\nabla \cdot \vec{v})^{2} d V
$$

Since we are still attempting a solution in the linear limit, the lowest-order contribution to the power dissipation must be second order in the wave's displacement from equilibrium; in this case, $T_{1}^{2}$ and $\left|\vec{v}_{1}\right|^{2}$, hence it is positive definite (see Sect. 10.5). For that reason, the absolute temperature, $T$, can be taken outside the integral and represented by $T_{m}$, since allowing for acoustical variation of that temperature term would add a correction to the thermal conduction loss that is third order in displacements from equilibrium.

For a plane wave propagating in the $x$ direction, it is convenient to express $v_{x}=v_{1} \sin (\omega t-k x)$, setting $v_{y}=v_{z}=0$. Substitution into the last two terms of Eq. (14.13) produces the (nonthermal) mechanical dissipation.

$$
-\left(\frac{4}{3} \mu+\zeta\right) \int\left(\frac{\partial v_{1}}{\partial x}\right)^{2} d V=-k^{2}\left(\frac{4}{3} \mu+\zeta\right) v_{1}^{2} \int \cos ^{2}(\omega t-k x) d V
$$

Since we are only interested in the time-averaged power dissipation, the contributions from the nonthermal terms in Eq. (14.13) is $-\left(k^{2} / 2\right)[(4 \mu / 3)+\zeta] v_{1}^{2} V_{o}$, where $V_{o}$ is the volume of the fluid under consideration through which the plane wave is propagating.

It is worth comparing the appearance of the factor, $4 / 3$, that multiplies the shear viscosity, $\mu$, with the corresponding expression for the modulus of unilateral compression, $D$ (aka the dilatational modulus), introduced in Sect. 4.2.2, to the shear modulus, $G$, and bulk modulus, $B$, in Table 4.1: $D=(4 G / 3)+B$. Again, this is a direct consequence of the fact that the distortion produced by a plane wave can be decomposed into two shears (related to $G$ ) and a hydrostatic compression (related to $B$ ).

The result in Eq. (14.14), without $\zeta$, was first produced by Stokes who expressed the result as the temporal attenuation coefficient [4]. The lack of agreement between his theoretical predictions and experimental measurements provided the starting point for the modern attempts to account for

\footnotetext{
${ }^{3}$ The component form assumes that the equation is summed over the repeated indices, $i$ and $k$. This is known as the "Einstein summation convention."
} 
attenuation in terms of molecular relaxation [5]. The spatial attenuation coefficient, due to viscous dissipation, was first introduced by Stefan in $1866^{4}$ [6]. The first calculation to include both the effects of thermal conductivity and shear viscosity on the absorption of sound was published by Kirchhoff in 1868 [7].

To evaluate the contribution of thermal conduction to the mechanical dissipation in Eq. (14.13), the temperature change needs to be related to the pressure change to evaluate the one-dimensional temperature gradient, $(\partial T / \partial x)$. For an ideal gas, this relation should be familiar, having been derived in Eqs. (1.21) and (7.25).

$$
\left(\frac{\partial T}{\partial p}\right)_{s}=\left(\frac{\gamma-1}{\gamma}\right) \frac{T_{m}}{p_{m}}
$$

Since we seek an attenuation coefficient that would be applicable to all fluids, a more general expression for $(\partial T / \partial p)_{s}$ needs to be calculated to evaluate $(\partial T / \partial x)$.

$$
\left(\frac{\partial T}{\partial x}\right)_{s}=\left(\frac{\partial T}{\partial p}\right)_{s}\left(\frac{\partial p}{\partial v}\right)_{s}\left(\frac{\partial v}{\partial x}\right)_{s}=-\rho_{m} c\left(\frac{\partial T}{\partial p}\right)_{s} k v_{1} \cos (\omega t-k x)
$$

The derivative of pressure with respect to velocity for a nearly adiabatic plane wave is a direct consequence of the Euler equation: $p_{1}=\rho_{m} c v_{1}$.

The derivative of temperature with respect to density can be evaluated using the enthalpy function, $H(S, p)=U+p V$, that sums the internal energy, $U$, introduced in Sect. 7.1.2 to calculate heat capacities, with the mechanical work, $W=p V$. From Eqs. (7.8, 7.9, and 7.10), the internal energy, $U(S$, $V)$, can be transformed into the enthalpy, $H(S, p)$, using the product rule for differentiation. In thermodynamics, this operation is known as a Legendre transformation [8].

$$
\begin{gathered}
d U=T d S-p d V=T d S-d(p V)+V d p \\
\Rightarrow d(U+p V) \equiv d H=T d S+V d p
\end{gathered}
$$

The change in enthalpy, $d H(S, p)$, can be expanded in a Taylor series, retaining only the linear terms.

$$
d H=\left(\frac{\partial H}{\partial S}\right)_{p} d S+\left(\frac{\partial H}{\partial p}\right)_{S} d p
$$

Comparison of Eqs. (14.17) and (14.18) can be used to evaluate those derivatives.

$$
\left(\frac{\partial H}{\partial S}\right)_{p}=T \quad \text { and } \quad\left(\frac{\partial H}{\partial p}\right)_{S}=V
$$

Since the order of differentiation is irrelevant, the mixed partial derivatives must be equal.

$$
\frac{\partial^{2} H}{\partial p \partial S}=\frac{\partial^{2} H}{\partial S \partial p} \Rightarrow\left(\frac{\partial T}{\partial p}\right)_{S}=\left(\frac{\partial V}{\partial S}\right)_{p}
$$

This result is one of several thermodynamic identities known as the Maxwell relations [9].

\footnotetext{
${ }^{4}$ Stefan was the thesis advisor of Boltzmann, who was the advisor of Ehrenfest, who was the advisor of Uhlenbeck, who was the advisor of Putterman, who was my advisor, along with Isadore Rudnick, when I was a graduate student at UCLA.
} 


$$
\left(\frac{\partial V}{\partial S}\right)_{p}=\left(\frac{\partial V}{\partial T}\right)_{p}\left(\frac{\partial T}{\partial S}\right)_{p}
$$

The result in Eq. (14.21) can be expressed in terms of tabulated material properties [10] using the definition of the (extensive) heat capacity at constant pressure, $C_{p}$, or the (intensive) specific heat (per unit mass) at constant pressure, $c_{p}$, from Eq. (7.14), and the definition of the isobaric (constant pressure) volume coefficient of thermal expansion, $\beta_{p}$.

$$
C_{p}=T\left(\frac{\partial S}{\partial T}\right)_{p} \quad \text { or } \quad c_{p}=T\left(\frac{\partial s}{\partial T}\right)_{p} \quad \text { and } \quad \beta_{p}=\frac{1}{V}\left(\frac{\partial V}{\partial T}\right)_{p}=-\frac{1}{\rho_{m}}\left(\frac{\partial \rho}{\partial T}\right)_{p}
$$

These results can be combined to produce an expression for the temperature gradient required to evaluate the thermal conduction integral in Eq. (14.13) using Eq. (14.16).

$$
\frac{\partial T}{\partial x}=c \frac{\beta_{p} T_{m}}{c_{p}} \frac{\partial v_{1}}{\partial x}=-c \frac{\beta_{p} T_{m}}{c_{p}} v_{1} k \cos (\omega t-k x)
$$

As before, our interest will be in the time-averaged value for evaluation of the thermal conduction term in the integral expression for $\Pi_{\text {mech }}$ in Eq. (14.12).

$$
\left\langle-\frac{\kappa}{T_{m}} \int(\nabla T)^{2} d V\right\rangle_{t}=\frac{-\kappa c^{2} \beta_{p}^{2} v_{1}^{2} k^{2}}{2 c_{p}^{2}} V_{o}
$$

This result can be evaluated in terms of the difference in the specific heats that was shown by thermodynamic arguments to be $C_{P}-C_{V}=\mathfrak{R}$, in Eq. (7.14), for an ideal gas, or $c_{p}-c_{v}=\mathfrak{R} / M$, where $\mathfrak{R}$ is the universal gas constant and $M$ is the mean molecular or atomic mass of the ideal gas or ideal gas mixture. By the same thermodynamic arguments, the general result for the specific heat difference can be expressed in terms of the fluid parameters in Eq. (14.22) [11].

$$
c_{p}-c_{v}=T \beta_{p}^{2}\left(\frac{\partial p}{\partial \rho}\right)_{T}=T \beta_{p}^{2}\left(\frac{c_{v}}{c_{p}}\right)\left(\frac{\partial p}{\partial \rho}\right)_{s}=T \beta_{p}^{2} c^{2}\left(\frac{c_{v}}{c_{p}}\right)
$$

The relationship between the square of the isothermal sound speed, $(\partial p / \partial \rho)_{T}$, and the square of the adiabatic sound speed, $(\partial p / \partial \rho)_{s}=c^{2}$, should be familiar since $\left(c_{v} / c_{p}\right) \equiv \gamma^{-1}$.

Substitution of Eq. (14.25) into Eq. (14.24) provides a compact expression for the time-averaged power dissipation that is valid for ideal gases as well as for all other homogeneous fluids.

$$
\left\langle-\frac{\kappa}{T_{m}} \int(\nabla T)^{2} d V\right\rangle_{t}=-(1 / 2) \kappa k^{2} v_{1}^{2} V_{o}\left(\frac{1}{c_{v}}-\frac{1}{c_{p}}\right)
$$

Combining Eq. (14.26) with Eq. (14.14) provides an expression for the time-averaged mechanical power dissipation due to all of the irreversible dissipation mechanisms.

$$
\left\langle\Pi_{\text {mech }}\right\rangle_{t}=-(1 / 2) k^{2} v_{1}^{2} V_{o}\left[\left(\frac{4}{3} \mu+\zeta\right)+\kappa\left(\frac{1}{c_{v}}-\frac{1}{c_{p}}\right)\right]
$$

The total energy, $E$, of the plane wave occupying the volume, $V_{o}$, can be expressed in terms of the maximum kinetic energy density, $(\mathrm{KE})_{\max }$. 


$$
E=(\mathrm{KE})_{\max } V_{o}=(1 / 2) \rho_{m} v_{1}^{2} V_{o}
$$

Since the decay rate of the energy is twice that of the amplitude decay rate, the spatial attenuation constant that reflects thermoviscous losses (and whatever $\zeta$ represents!), $\alpha_{T-V}$, can be written in terms of the time-averaged power dissipation, $\left\langle\Pi_{m e c h}\right\rangle_{t}$, in Eq. (14.27), and the average total energy, $E$, in Eq. (14.28).

$$
\alpha_{T-V}=\frac{\left|\left\langle\Pi_{m e c h}\right\rangle_{t}\right|}{2 c E}=\frac{\omega^{2}}{2 \rho_{m} c^{3}}\left[\left(\frac{4}{3} \mu+\zeta\right)+\kappa\left(\frac{1}{c_{v}}-\frac{1}{c_{p}}\right)\right]
$$

This final result for $\alpha_{T-V}$ is valid for all fluids as long as the decrease in the sound wave's amplitude over the distance of a single wavelength is relatively small, $\alpha_{T-V} \lambda \ll 1$, since the stored energy was calculated for an undamped sound wave.

We also see that this result is similar to the "almost correct" result, $\alpha_{\text {almost }}$, calculated from the Navier-Stokes equation in Sect. 14.1. The dependence on frequency, $\omega$; mass density, $\rho_{m}$; and sound speed, $c$, is identical, but the shear viscosity, $\mu$, is no longer the only transport property of the medium that contributes to attenuation of the sound wave; in Eq. (14.29), $\mu$ has been replaced by the term within the square bracket.

As demonstrated earlier in Eq. (14.8), the expression for attenuation given in Eq. (14.29) will always be valid for sound in gases, since the kinematic viscosity, $\nu_{\text {gas }}=\mu_{\text {gas }} / \rho_{m}$, is on the order of the product of the mean free path, $\bar{\ell}$, times the mean thermal velocity of the gas molecules or the sound speed.

$$
\frac{\nu_{\text {gas }} \omega}{c^{2}} \cong \bar{\ell} \frac{\omega}{c} \cong \frac{\bar{\ell}}{\lambda} \ll 1
$$

\subsection{Classical Thermoviscous Attenuation}

Before the role of molecular relaxation was appreciated and the associated dissipative coefficient, $\zeta$, was introduced, attenuation of sound due to thermoviscous losses was calculated by Kirchhoff [7]. That result is often called the classical absorption coefficient, $\alpha_{\text {classical }}$.

$$
\alpha_{\text {classical }}=\frac{\omega^{2}}{2 \rho_{m} c^{3}}\left[\frac{4}{3} \mu+\kappa\left(\frac{1}{c_{v}}-\frac{1}{c_{p}}\right)\right]=\frac{\omega^{2}}{2 c^{3}}\left[\frac{4}{3} \frac{\mu}{\rho_{m}}+\frac{\kappa}{\rho_{m} c_{p}}\left(\frac{c_{p}}{c_{v}}-1\right)\right]
$$

For an ideal gas, the classical attenuation coefficient can be expressed more transparently in terms of the kinematic viscosity, $\nu=\mu / \rho_{m}$; the polytropic coefficient, $\gamma=c_{p} / c_{v}$; and the dimensionless ratio of the thermal and viscous diffusion constants, known as the Prandtl number, $\operatorname{Pr} \equiv\left(\mu / c_{p} \kappa\right)=\left(\delta_{\nu} / \delta_{\kappa}\right)^{2}$, that was introduced in Sect. 9.5.4.

$$
\alpha_{\text {classical }}=\frac{\omega^{2} \nu}{2 c^{3}}\left[\frac{4}{3}+\frac{(\gamma-1)}{\operatorname{Pr}}\right] \Rightarrow \frac{\alpha_{\text {classical }}}{f^{2}}=\frac{2 \pi^{2} \nu}{c^{3}}\left[\frac{4}{3}+\frac{(\gamma-1)}{\operatorname{Pr}}\right]
$$

Most single-component gases and many gas mixtures have $\operatorname{Pr} \cong 2 / 3$. For air at atmospheric pressure and $20{ }^{\circ} \mathrm{C}, \nu=1.51 \times 10^{-5} \mathrm{~m}^{2} / \mathrm{s}, \operatorname{Pr}=0.709, \gamma=1.402$, and $c=343.2 \mathrm{~m} / \mathrm{s}$. Under those conditions, $\alpha_{\text {classical }} / f^{2}=1.40 \times 10^{-11} \mathrm{~s}^{2} / \mathrm{m}$. The accepted value of $\alpha / f^{2}$ in the high-frequency limit is $1.84 \times 10^{-11} \mathrm{~s}^{2} / \mathrm{m}$. This discrepancy is due to the absence of $\zeta$ in Eq. (14.32) [12]. 
Since the difference between the specific heats at constant pressure and constant volume is small for liquids, the viscous contribution to the classical attenuation constant is dominant. For pure water at $280 \mathrm{~K}, \nu_{\mathrm{H}_{2} \mathrm{O}}=1.44 \times 10^{-6} \mathrm{~m}^{2} / \mathrm{s}$ and $\operatorname{Pr}_{\mathrm{H}_{2} \mathrm{O}}=10.4$, [13] with $c_{\mathrm{H}_{2} \mathrm{O}}=1500 \mathrm{~m} / \mathrm{s}$, making $\alpha_{\text {classical }} / f$ $2=1.1 \times 10^{-14} \mathrm{~s}^{2} / \mathrm{m}$ for freshwater. The accepted value of $\alpha / f^{2}$ in the high-frequency limit is $2.5 \times 10^{-14} \mathrm{~s}^{2} / \mathrm{m}$, again due to the absence of $\zeta$ in Eq. (14.32) [14].

\subsection{The Time-Dependent Equation of State}

The distortion of a fluid element caused by passage of a plane wave was decomposed into shear deformations, which changed the shape of the element, and a hydrostatic deformation, which changed the volume of the element, as diagrammed schematically in Fig. 14.1. Each of those deformations introduced irreversibility that increased entropy as expressed in Eq. (14.12), leading to energy dissipation as expressed in Eq. (14.13). Based on the discussion in Sect. 9.4, the shear viscosity, $\mu$, was introduced to relate the shear deformations to the dissipative shear stresses.

Another coefficient, $\zeta$, was introduced to relate entropy production to the divergence of the fluid's velocity field, $\nabla \cdot \vec{v}$. The continuity equation requires that when $\nabla \cdot \vec{v} \neq 0$, the density of the fluid must also be changing: $(\partial \rho / \partial t) \neq 0$. Why should a change in the fluid's density be related to irreversible entropy production?

When the phenomenological model was introduced, it was assumed that only five variables were required to completely specify the state of a homogeneous, isotropic, single-component fluid: one mechanical variable ( $p$ or $\rho$ ) and one thermal variable ( $s$ or $T$ ), along with the three components of velocity (e.g., $v_{x}, v_{y}$, and $v_{z}$ ). For a static fluid, $|v|=0$, only two variables were required, resulting in the laws of equilibrium thermodynamics (i.e., energy conservation and entropy increase) rather than the laws of hydrodynamics (that also incorporate thermodynamics). The evolution of those variables was determined by the imposition of five conservation equations (i.e., mass, entropy, and vector momentum). That assertion included an implicit assumption that an equation of state existed and it could be used to relate the thermodynamic variables (mechanical and thermal) to each other instantaneously.

For some fluids, the assumption of an instantaneous response of the density to changes in the pressure is not valid. (Noble gases are one notable exception, since they do not have any rotational degrees of freedom.) The microscopic models of gases that were based on the kinetic theory introduced the concept of collision times between the constituent particles (atoms and/or molecules) and the Equipartition Theorem in Eq. (7.2) that stated that through these collisions, an equilibrium could be established that distributed the total thermal energy of the system equitably (on average) among all of the available degrees of freedom. What has been neglected (to this point) was the fact that the collisions take a non-zero time to establish this equilibrium; if the conditions of the fluid element are changing during this time, the system might never reach equilibrium.

How did we get away with this "five-variable fraud" for so long? One answer is hidden in the transition from adiabatic sound speed in an ideal gas to the isothermal sound speed. Equation (9.24) defined a critical frequency, $\omega_{\text {crit }}$, at which the speed of thermal diffusion was equal to the speed of sound propagation. At that frequency, the wavelength of sound corresponded to a distance, which was about 20 times the average spacing between particles, known as the mean free path between collisions, $\bar{\ell}$. Since that collision frequency was so much higher than our frequencies of interest, the equilibration between translational and rotational degrees of freedom in gases of polyatomic molecules occurred so quickly that the equation of state appeared to act instantaneously [15]. Even though a vibrating object couples to the translational degrees of freedom in a gas, the translational and rotational degrees of freedom came into equilibrium in much less time than the period of the vibrating object's oscillations. 
Fig. 14.2 Relaxation frequencies, $f_{R}=\left(2 \pi \tau_{R}\right)^{-1}$, for equilibration between $\mathrm{O}_{2}$ or $\mathrm{N}_{2}$ and water vapor $\left(\mathrm{H}_{2} \mathrm{O}\right)$ as a function of the mole fraction of water vapor in air, $h$, at $p_{m}=1$ atmosphere. At the top of the graph are scales that can be used to relate the mole fraction of $\mathrm{H}_{2} \mathrm{O}$ on the $x$ axis to the more popular designation of percent "relative humidity" at $5{ }^{\circ} \mathrm{C}$ and $20{ }^{\circ} \mathrm{C}[16]$

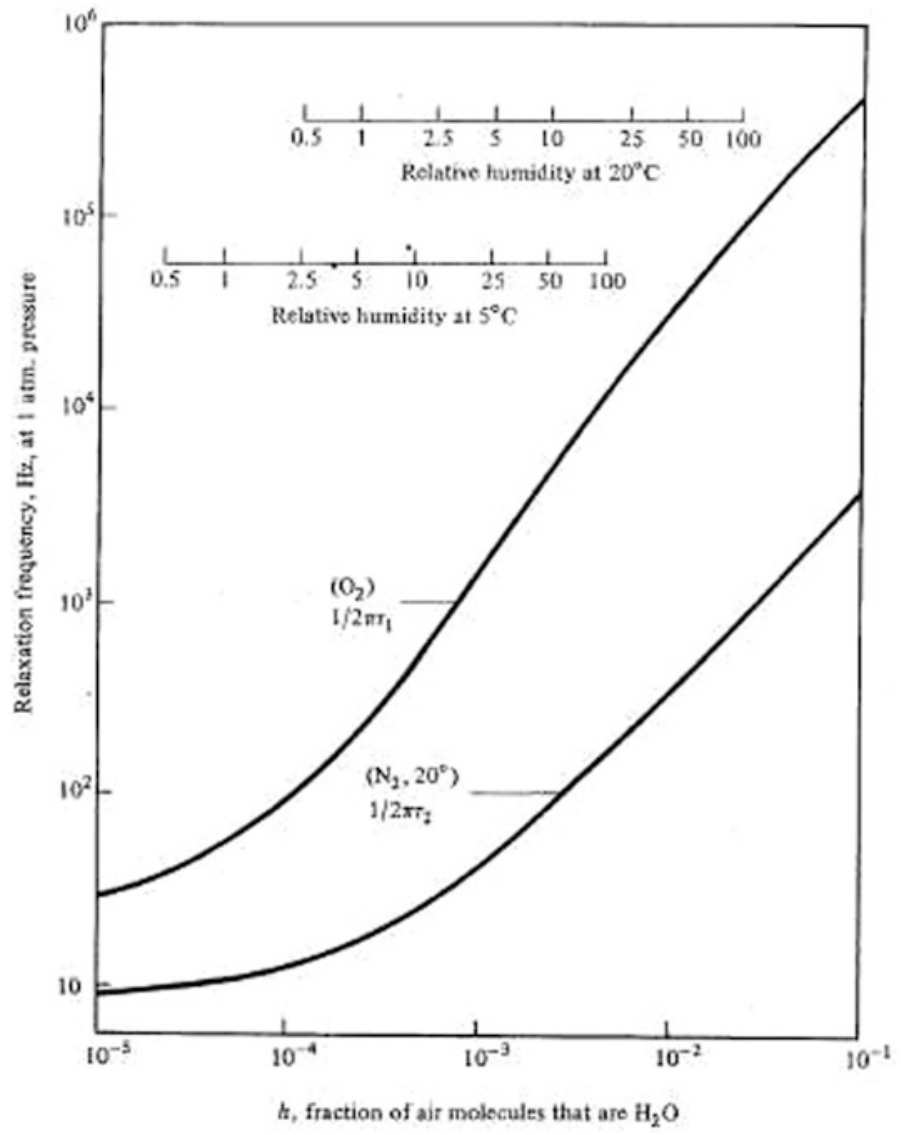

That "fraud" was obscured by our use of $\gamma=7 / 5$ in the expression for sound speed which treated the air as instantly sharing energy between the internal translational and rotational degrees of freedom.

When there are other components in a gas or liquid, they may have relaxation times that are sufficiently close to the acoustic periods of interest that their "equilibration" to the acoustically induced changes cannot be considered to occur instantaneously. In air, for example, if there is water vapor present, it will equilibrate with the $\mathrm{O}_{2}$ and $\mathrm{N}_{2}$ over times that are comparable to the periods of sound waves of interest for human perception (i.e., $20 \mathrm{~Hz} \lesssim f \lesssim 20 \mathrm{kHz}$ ). Figure 14.2 shows the relaxation frequencies as a function of the mole fraction, $h$, of $\mathrm{H}_{2} \mathrm{O}$ and also relative humidity as a percentage [16].

Those equilibration times are dependent upon the gas mixture's temperature, pressure, and mixture concentration (i.e., mole fraction of water vapor, $h$, or relative humidity, $R H$ ) [17]. It is apparent that the relaxation frequencies in Fig. 14.2 are in the audio range for ordinary values of temperature and humidity [17].

$$
\begin{aligned}
& f_{r O}=\frac{p_{m}}{p_{\text {ref }}}\left\{24+\left[\frac{\left(4.04 \times 10^{4} h\right)(0.02+h)}{0.391+h}\right]\right\} \\
& f_{r N}=\frac{p_{m}}{p_{\text {ref }}}\left(\frac{T}{T_{\text {ref }}}\right)^{-1 / 2}\left(9+280 h \exp \left\{-4.170\left[\left(\frac{T}{T_{\text {ref }}}\right)^{-1 / 3}-1\right]\right\}\right)
\end{aligned}
$$


The relaxation frequencies for water vapor and nitrogen, $f_{r N}$, and for water vapor and oxygen, $f_{r O}$, assume a standard atmospheric composition with $78.1 \%$ nitrogen, $20.9 \%$ oxygen, and $314 \mathrm{ppm}$ carbon dioxide at a reference pressure, $p_{r e f}=101,325 \mathrm{~Pa}$, and reference temperature, $T_{r e f}=293.15 \mathrm{~K}=20.0^{\circ} \mathrm{C}$. In Eq. (14.33), the molar concentration of water vapor, $h$, is expressed in percent. For ordinary atmospheric conditions near sea level, $0.2 \% \lesssim h \lesssim 2.0 \%$.

To relate $R H$ to $h$ (both in \%), it is first necessary to calculate the saturated vapor pressure of water in air, $p_{\text {sat }}$, relative to ambient pressure, $p_{\text {ref }}=101.325 \mathrm{kPa}$, using the triple-point isotherm temperature, $T_{01}=273.16 \mathrm{~K}=+0.01{ }^{\circ} \mathrm{C}$.

$$
\frac{p_{\text {sat }}}{p_{\text {ref }}}=10^{C} ; \quad C=-6.8346\left(\frac{T_{01}}{T}\right)^{1.261}+4.6151
$$

The molar concentration of water vapor, $h$, in percent, can then be expressed in terms of the relative humidity, $R H$, also in percent [17].

$$
h=R H\left(\frac{p_{s a t}}{p_{m}}\right)
$$

A similar effect is observed in seawater where boric acid, $\mathrm{B}(\mathrm{OH})_{3}$, and magnesium sulfate, $\mathrm{MgSO}_{4}$, have relaxation frequencies of $1.18 \mathrm{kHz}$ and $145 \mathrm{kHz}$, respectively, at $20^{\circ} \mathrm{C}$ [18]. In the case of these salts, the relaxation time represents the pressure-dependent association-dissociation reaction between the dissolved salts and their ions.

\subsection{Attenuation due to Internal Relaxation Times}

"If a system is in stable equilibrium, then any spontaneous change of its parameters must bring about processes which tend to restore the system to equilibrium." H. L. Le Châtelier ${ }^{5}$

A new positive scalar coefficient, $\zeta$, was introduced in the entropy conservation Eq. (14.12) to scale the irreversibility of hydrostatic fluid deformations. It has the same units as the shear viscosity [Pa-s $]^{6}$ and is usually of about the same magnitude. If the medium does not possess any additional internal degrees of freedom that have to be brought into equilibrium, then its value can be identically zero. That constant is zero for the noble gases ( $\mathrm{He}, \mathrm{Ne}, \mathrm{Ar}, \mathrm{Kr}, \mathrm{Xe}$, and $\mathrm{Rn}$ ) that are intrinsically monatomic with atoms that are spherically symmetrical, thus lacking rotational degrees of freedom (see Sect. 7.2). On the other hand, as suggested in Fig. 14.2, if there are processes with relaxation times that are near the frequencies of interest, the value of $\zeta$ can be orders of magnitude greater than $\mu$ near those frequencies.

With acoustical compressions or expansions, as in any rapid change of state, the fluid cannot remain in thermodynamic equilibrium. Following Le Chatlier's Principle, ${ }^{5}$ the system will attempt to return to a new equilibrium state that is consistent with the new parameter values that moved it away from its

\footnotetext{
${ }^{5}$ Henry Louis Le Châtelier (1850-1936) was a Parisian chemist. This principle is sometimes also attributed to German physicist Karl Ferdinand Braun (1850-1918), the inventor of the cathode-ray tube and the oscilloscope.

${ }^{6}$ Because $\zeta$ has the same units as shear viscosity, it is commonly called bulk viscosity or second viscosity, even though its effects are entirely unrelated to shear strains. I find both terms misleading and attempt to avoid their use in this textbook, although acousticians have to be aware that they represent the common nomenclature used to identify losses related to the relaxation of internal degrees of freedom within fluids.
} 
previous state of equilibrium. In some cases, this equilibration takes place very quickly so that the medium behaves as though it were in equilibrium at all times. In other cases, the equilibration is slow, and the medium never catches up. In either case, the processes that attempt to reestablish equilibrium are irreversible and therefore create entropy and dissipate energy.

If $\xi$ represents some physical parameter of the fluid and $\xi_{o}$ represents the value of $\xi$ at equilibrium, then if the fluid is not in equilibrium, $\xi$ will vary with time. If the fluid is not too far from equilibrium, so the difference, $\xi-\xi_{o}$, is small (i.e., $\left|\xi-\xi_{o}\right| / \xi_{o} \ll 1$ ), and then the rate of change of that parameter, $\dot{\xi}$, can be expanded in a Taylor series retaining only the first term and recognizing that any zero-order contribution to $\dot{\xi}$ must vanish since $\xi=\xi_{o}$ at equilibrium.

$$
\dot{\xi}=-\frac{\left(\xi-\xi_{o}\right)}{\tau_{R}}
$$

This suggests an exponential relaxation of the system toward its new equilibrium state. Le Châtelier's Principle requires that the rate must be negative and that the relaxation time, $\tau_{R}$, must be positive.

For acoustically induced sinusoidal variations in the parameter, $\xi$, at frequency, $\omega$, the sound speed will depend upon the relative values of the period of the sound, $T=2 \pi / \omega$, and the relaxation time, $\tau_{R}$. If the period of the disturbance is long compared to the exponential equilibration time, $\tau_{R}$, so that $\omega \tau_{R}=2 \pi \tau_{R} / T \ll 1$, then the fluid will remain nearly in equilibrium at all times during the acoustic disturbance. In that limit, the sound speed will be the equilibrium sound speed, $c_{o}$. In the opposite limit, $\omega \tau_{R}=2 \pi \tau_{R} / T \gg 1$, the medium's sound speed, $c_{\infty}>c_{o}$, will be determined by the fluid's elastic response if the internal degrees of freedom cannot be excited by the disturbance. Said another way, the internal degrees of freedom are "frozen out" in that limit; they simply do not have enough time to participate before the state of the system has changed.

One way to think about this effect is to consider the sound speed in a gas of diatomic molecules that possess three translational degrees of freedom and two rotational degrees of freedom. The specific heats of monatomic and polyatomic gases were discussed in Sect. 7.2, and the relationship between the sound speed in such gases and the specific heat ratio, $\gamma=c_{p} / c_{v}$, is provided in Eq. (10.22). If the rotational degrees of freedom are not excited, then the gas behaves as though it were monatomic, so $\gamma=5 / 3$. If the rotational and translational degrees of freedom are always in equilibrium, then $\gamma=7 / 5<5 / 3$, so $c_{\infty}=\sqrt{25 / 21} c_{o}$.

The mathematical "machinery" needed to represent the attenuation and dispersion of sound waves in a homogeneous medium with an internal degree of freedom, or a "relaxing sixth variable," has already been developed to describe viscoelastic solids in Sect. 4.4.2. Figure 4.25 could just as well describe the propagation speed (solid line) as a function of the nondimensional frequency, $\omega \tau_{R}$, with $c_{\mathrm{o}}$ being the limiting sound speed for $\omega \tau_{R}=2 \pi \tau_{R} / T \ll 1$ and $c_{\infty}$ being the sound speed for $\omega \tau_{R}=2 \pi \tau_{R} /$ $T \gg 1$. In addition, the Kramers-Kronig relations of Sect. 4.4 .4 would still apply; the variation in sound speed with frequency requires a frequency-dependent attenuation, shown in Fig. 4.25 as the dashed line, and vice versa.

The transformation of the results derived for the stiffness and damping of a viscoelastic medium simply requires that the sound speed is proportional to the square root of the elastic modulus (i.e., stiffness) as expressed in Eq. (10.21). That substitution allows Eq. (4.67) to produce the propagation speed as a function of the nondimensional frequency, $\omega \tau_{R}$.

$$
c^{2}=c_{o}^{2}+\left(c_{\infty}^{2}-c_{o}^{2}\right) \frac{\left(\omega \tau_{R}\right)^{2}}{1+\left(\omega \tau_{R}\right)^{2}}
$$

The same approach applied to Eq. (4.70) provides the attenuation per wavelength, $\alpha \lambda$, as function of the dimensionless frequency, $\omega \tau_{R}$. 


$$
(\alpha \lambda)=2 \pi \frac{\left(c_{\infty}^{2}-c_{o}^{2}\right)\left(\omega \tau_{R}\right)}{c_{o}^{2}\left[1+\left(\omega \tau_{R}\right)^{2}\right]+\left(c_{\infty}^{2}-c_{o}^{2}\right)\left(\omega \tau_{R}\right)^{2}}
$$

Following Eq. (4.71) or Eq. (4.89), the maximum value of attenuation per wavelength in Eq. (14.38) will occur at a unique value of the nondimensional frequency, $\left(\omega \tau_{R}\right)_{\max }$.

$$
\left(\omega \tau_{R}\right)_{\max }=\frac{c_{o}}{c_{\infty}} \quad \text { and } \quad(\alpha \lambda)_{\max }=\pi \frac{\left(c_{\infty}^{2}-c_{o}^{2}\right)}{c_{\infty} c_{o}}
$$

The consequence of the Kramers-Kronig relations for such single relaxation time phenomena, as emphasized in Sect. 4.4.2, is that the attenuation is entirely determined by the dispersion, and vice versa.

Using these results, it is possible to write simple universal expressions for attenuation due to excitation of internal degrees of freedom in terms of the relaxation frequency, $f_{R}=\left(2 \pi \tau_{R}\right)^{-1}$.

$$
\frac{\alpha \lambda}{(\alpha \lambda)_{\max }}=\frac{2}{\frac{f}{f}+\frac{f}{f_{R}}} \Rightarrow \alpha(f)=\left[\frac{2(\alpha \lambda)_{\max }}{c f_{R}}\right] \frac{f^{2}}{1+\left(\frac{f}{f_{R}}\right)^{2}}
$$

The variation in the attenuation per wavelength, $\alpha \lambda$, and the propagation speed, $c$, as a function of nondimensional frequency, $\omega \tau_{R}$, is plotted in Fig. 14.3 and should be compared to the plot for a viscoelastic solid in Fig. 4.25, which exhibits identical behavior. For relaxation frequencies, $f_{R}$, that are much higher than the frequency of interest, $f$, the attenuation constant's quadratic frequency dependence is recovered, as was derived in Eq. (14.7) for $\alpha_{\text {almost }}$ and in Eq. (14.29) for $\alpha_{T-V}$.

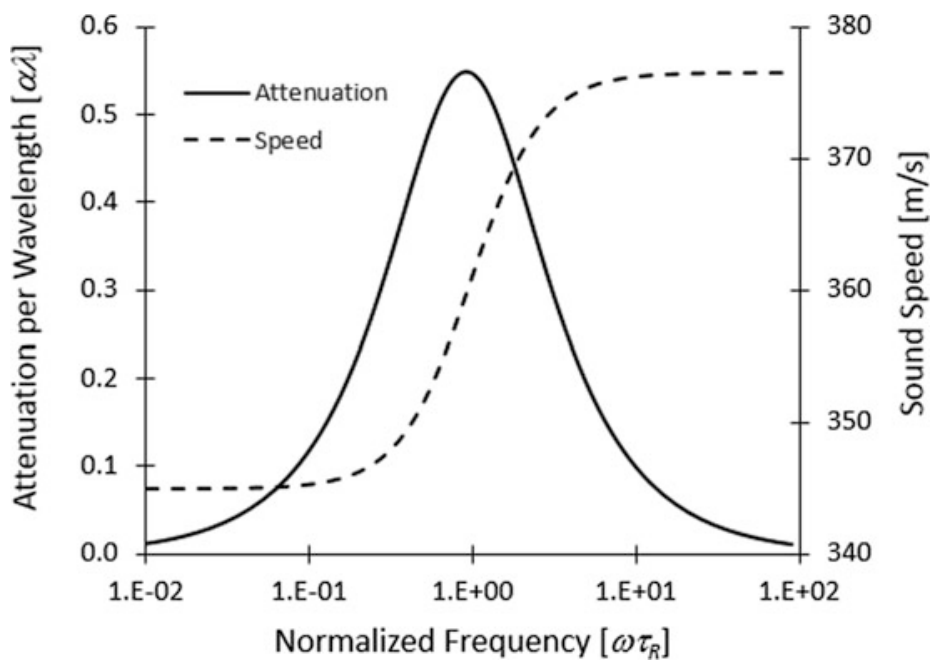

Fig. 14.3 The attenuation and dispersion for a fluid with $c_{\infty}=c_{o} \sqrt{25 / 21}$, where $c_{o}=345 \mathrm{~m} / \mathrm{s}$, as a function of the nondimensional frequency, $\omega \tau_{R}$. Values for the attenuation per wavelength (solid line), $\alpha \lambda$, should be read from the lefthand vertical axis, and the values of sound speed (dashed line) should be read from the right-hand axis. This behavior is identical to that of a viscoelastic solid that is shown in Fig. 4.25 since the Kramers-Kronig relations dictate the relationship between the real and imaginary parts of the linear response 


\subsubsection{Relaxation Attenuation in Gases and Gas Mixtures}

The first example of the effects of the relaxation of an internal degree of freedom on sound speed and attenuation in gas is taken from the measurements of Shields in fluorine [19]. Halogen vapors (e.g., chlorine, fluorine, bromine, iodine) are unique in that they consist of homonuclear diatomic molecules that have appreciable vibrational energy, $E_{V}=(n+1 / 2) \hbar \omega_{V}$, at room temperature (see Sect. 7.2.2). Because the diatomic molecules behave as simple (quantum mechanical) harmonic oscillators, that internal degree of freedom can be characterized by a single relaxation time corresponding to the radian period of their harmonic oscillations.

Figure 14.4 shows the measured values of attenuation per wavelength, $\alpha \lambda$, and sound speed, $c$, as a function of frequency (also normalized by pressure), in units of $\mathrm{kHz} / \mathrm{atm}$., for fluorine gas

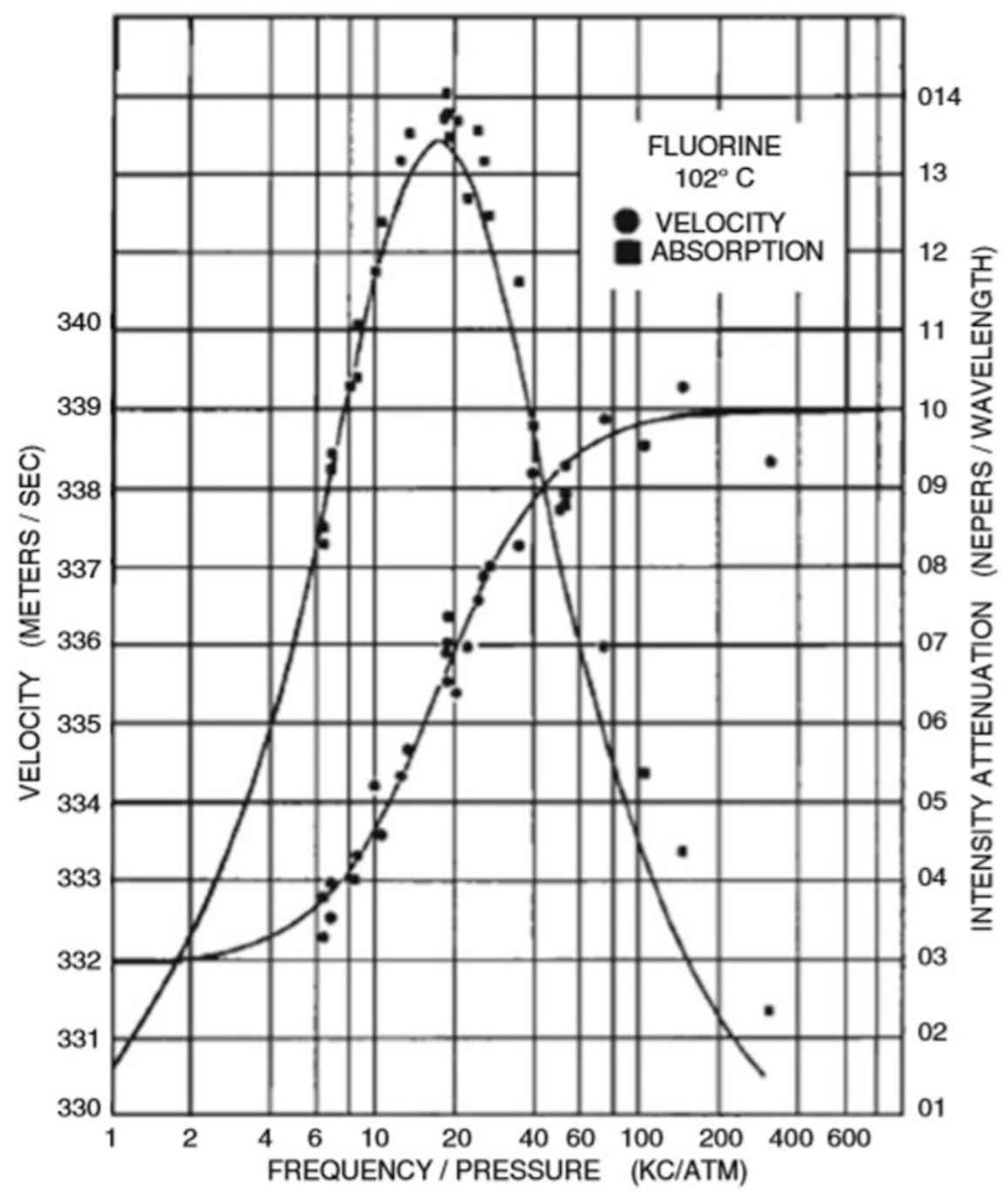

Fig. 14.4 Measured data for attenuation per wavelength and dispersion (sound speed) in fluorine gas at $102 \pm 2{ }^{\circ} \mathrm{C}$ [19]. The sound speed should be read from the left-hand vertical axis, and the attenuation per wavelength, $\alpha \lambda$, should be read from the right-hand axis that is labeled "Intensity Attenuation (Nepers/Wavelength)." Nepers is an archaic dimensionless unit that was in common usage at the time this data was published to designate $\alpha$ in Np/m. It simply refers to the spatial attenuation coefficient, named after John Napier, the inventor of logarithms. $1 \mathrm{~Np}=8.69 \mathrm{~dB}$. The solid lines are fits to the data points that are based on expressions like Eqs. (14.37) and (14.38) 
at $102 \pm 2{ }^{\circ} \mathrm{C}$, after correction for boundary layer losses at the surface of the tube containing the gas [20].

The vibrational relaxation time for diatomic fluorine at $102{ }^{\circ} \mathrm{C}$ is $\tau_{R}=10.7 \mu \mathrm{s}$, corresponding to a relaxation frequency, $f_{R}=\left(2 \pi \tau_{R}\right)^{-1}=14.9 \mathrm{kHz}$. Based on the fit to the sound speed, the limiting speeds are $c_{o}=332 \mathrm{~m} / \mathrm{s}$ and $c_{\infty}=339 \mathrm{~m} / \mathrm{s}$. The peak in the attenuation per wavelength, $(\alpha \lambda)_{\max }=0.13$, occurs at $\left(\omega \tau_{R}\right)_{\max }=0.98$, based on Eq. (14.39), in excellent agreement with the data in Fig. 14.4.

The relaxation attenuation in humid air is more complicated since the two relaxation frequencies for equilibration of the water vapor with the nitrogen and with the oxygen are different, as expressed in Eq. (14.33) and plotted in Fig. 14.2. Since energy loss is cumulative, it is possible to express the attenuation constant, $\alpha_{t o t}$, as the sum of the attenuation caused by the classical value, $\alpha_{\text {classical }}$, and the contributions from the two relaxation processes.

$$
\alpha_{\text {tot }}=\alpha_{\text {classical }}+\alpha_{O_{2}}+\alpha_{N_{2}}
$$

The pressure, frequency, and temperature dependence for the total attenuation coefficient is provided in combination with the relaxation times of Eq. (14.33) and plotted as a function of the frequency/pressure ratio for various values of the relative humidity in Fig. 14.5 [21].

$$
\begin{aligned}
\frac{\alpha_{\text {Air }}}{f^{2}} & =1.84 \times 10^{-11}\left(\frac{p_{m}}{p_{r e f}}\right)^{-1}\left(\frac{T}{T_{r e f}}\right)^{1 / 2}+\left(\frac{T}{T_{r e f}}\right)^{-5 / 2} \\
& \times\left\{0.01275 e^{-2,239 / T}\left[\frac{f_{r O}}{f_{r O}^{2}+f^{2}}\right]+0.1068 e^{-3,352 / T}\left[\frac{f_{r N}}{f_{r N}^{2}+f^{2}}\right]\right\}
\end{aligned}
$$

The difference between the classical attenuation constant and the total is clearly very large. At $2 \mathrm{kHz}$ and $1 \mathrm{~atm}$., $\alpha_{\text {classical }}=0.02 \mathrm{~dB} / \mathrm{m}$, but with $10 \%$ relative humidity, the attenuation is $\alpha_{\text {tot }}=0.80 \mathrm{~dB} / \mathrm{m}$.

Values for the attenuation in $\mathrm{dB} / \mathrm{km}$ are tabulated in a standard for different values of temperature from $-25{ }^{\circ} \mathrm{C}$ to $+50{ }^{\circ} \mathrm{C}$ and $10 \% \leq R H \leq 100 \%$ for pure tones with frequencies from $50 \mathrm{~Hz}$ to $10 \mathrm{kHz}$ in $1 / 3$-octave increments [17]. A small subset of that data are presented in Table 14.1.

\subsubsection{Relaxation Attenuation in Fresh and Salt Water}

As earlier noted in Sect. 14.3, the measured attenuation of sound in water is greater than $\alpha_{\text {classical }} / f^{2}$ based on the shear viscosity by more than a factor of two. In the calculation of $\alpha_{\text {classical }}$ for water, the thermal conductivity was neglected. It can be shown that neglect of the thermal conductivity is not the cause of this discrepancy. Measurements of attenuation at $4{ }^{\circ} \mathrm{C}$, where water has its density maximum and the thermal expansion coefficient vanishes, mean that $c_{p}=c_{v}$, so according to Eq. (14.25), there are no temperature changes associated with the acoustical pressure changes [22].

The excess attenuation has been ascribed to a structural relaxation process wherein a molecular rearrangement is caused by the acoustically produced pressure changes. During acoustic compression, the water molecules are brought closer together and are rearranged by being repacked more closely. This repacking takes a non-zero amount of time and leads to relaxational attenuation that makes $\zeta \neq 0$ [23]. The relaxation time as a function of water temperature for this process, $\tau_{R}$, is on the order of picoseconds and is plotted as a function of temperature in Fig. 14.6.

At $4{ }^{\circ} \mathrm{C}, \tau_{R} \cong 3.5 \mathrm{ps}$, corresponding to a relaxation frequency, $f_{R}=\left(2 \pi \tau_{R}\right)^{-1}=45 \mathrm{GHz}$, well above experimentally accessible frequencies. For that reason, there are no "relaxation bumps" in the attenuation vs. frequency, as seen in Fig. 14.7. Nonetheless, this structural relaxation makes $\zeta>\mu$, accounting for the excess attenuation in pure water. 


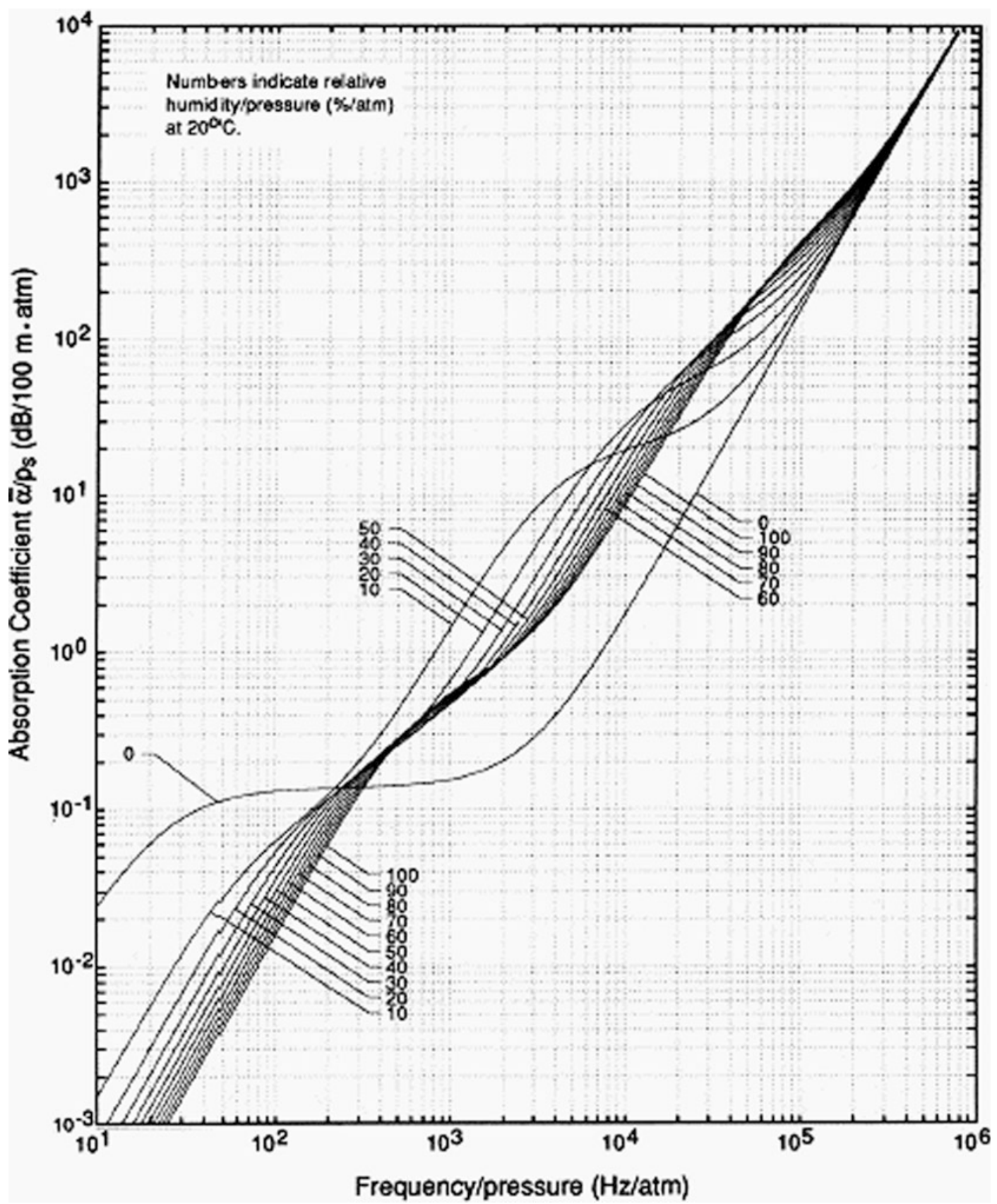

Fig. 14.5 Sound absorption coefficient per atmosphere in air at $20{ }^{\circ} \mathrm{C}$. The parameter labeling the individual curves is the relative humidity from $0 \%$ to $100 \%$. [21] The additional attenuation for dry air $(R H=0)$ is due to collisions between $\mathrm{N}_{2}$ and $\mathrm{CO}_{2}$

The attenuation of sound in seawater is similar to that in humid air where the relaxation frequencies are within a frequency range of interest. In seawater, there are two pressure-dependent ionic association-dissociation reactions due to the dissolved boric acid, $\mathrm{B}(\mathrm{OH})_{3}$, and the dissolved magnesium sulfate, $\mathrm{MgSO}_{4}$. Their contributions to the attenuation have the generic form introduced in Eq. (14.40). 
Table 14.1 (Left) Attenuation in $\mathrm{dB} / \mathrm{km}$ for air at $20^{\circ} \mathrm{C}$ with $R H=50 \%$, as a function of frequency. (Right) Attenuation in $\mathrm{dB} / \mathrm{km}$ at $4.0 \mathrm{kHz}$, for air at $20^{\circ} \mathrm{C}$, in the relative humidity range of $20 \% \leq R H \leq 70 \%$ [17]

\begin{tabular}{l|l|l|l}
\hline Freq. $[\mathrm{kHz}]$ & {$[\mathrm{dB} / \mathrm{km}]$} & $\mathrm{RH}(\%)$ & {$[\mathrm{dB} / \mathrm{km}]$} \\
\hline 1.60 & 7.37 & 20 & 74.4 \\
\hline 2.00 & 9.85 & 30 & 48.5 \\
\hline 2.50 & 13.7 & 40 & 36.1 \\
\hline 3.15 & 19.8 & 50 & 29.4 \\
\hline 4.00 & 29.4 & 60 & 25.4 \\
\hline 5.00 & 44.4 & 70 & 22.9 \\
\hline 6.30 & 67.8 & & \\
\hline 8.00 & 104 & & \\
\hline 10.00 & 159 & &
\end{tabular}

Fig. 14.6 Structural relaxation time for the repacking of water molecules caused by acoustical pressure changes [23]

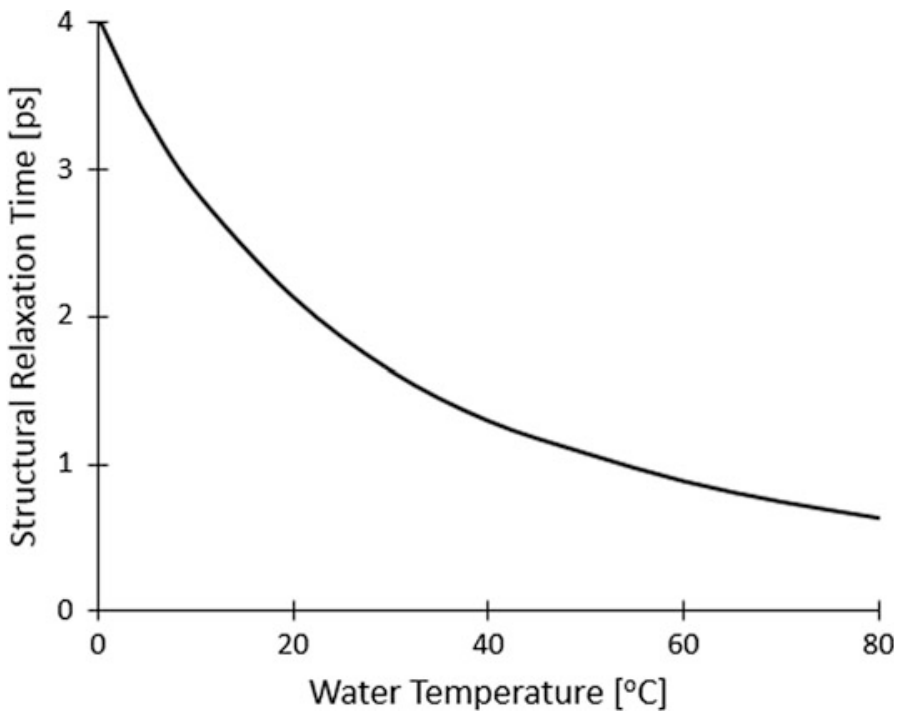

$$
\begin{array}{r}
\mathrm{MgSO}_{4}+\mathrm{H}_{2} \mathrm{O} \leftrightarrow \mathrm{Mg}^{+3}+\mathrm{SO}_{4}^{-2}+\mathrm{H}_{2} \mathrm{O} ; \alpha_{\mathrm{MgSO}_{4}} \cong \frac{4.6 \times 10^{-3}(\mathrm{kHz})^{2}}{4100+(\mathrm{kHz})^{2}} \\
\mathrm{~B}(\mathrm{OH})_{3}+(\mathrm{OH})^{-1} \leftrightarrow \mathrm{B}(\mathrm{OH})_{4}^{-1} ; \alpha_{\mathrm{B}(\mathrm{OH})_{3}} \cong \frac{1.2 \times 10^{-5}(\mathrm{kHz})^{2}}{1+(\mathrm{kHz})^{2}}
\end{array}
$$

The approximate attenuation values, $\alpha_{\mathrm{MgSO}_{4}}$ and $\alpha_{B(\mathrm{OH})_{3}}$, in Eq. (14.43) are in units of [ $\mathrm{m}^{-1}$ ] when the frequency is expressed in $\mathrm{kHz}$.

Those reactions that have relaxation frequencies that depend upon absolute temperature, $T$, and salinity, $S$, that is expressed in parts per thousand, \%o, and those relaxation frequencies, $f_{\mathrm{rBH}_{3} \mathrm{O}_{3}}$ and $f_{\mathrm{rMgSO}_{4}}$, are in hertz [24, 25].

$$
\begin{aligned}
f_{\mathrm{rBH}_{3} \mathrm{O}_{3}} & =2,800 \sqrt{\mathrm{S} / 35} \times 10^{[4-(1,245 / T)]} \\
f_{\mathrm{rMfSO}_{4}} & =\frac{8,170 \times 10^{[8-(1,990 / T)]}}{1+0.008(\mathrm{~S}-35)}
\end{aligned}
$$

For salinity, $\mathrm{S}=35 \%$, and $T=293 \mathrm{~K}, f_{\mathrm{rB}(\mathrm{OH})_{3}}=1.58 \mathrm{kHz}$ and $f_{\mathrm{rMgSO}_{4}}=132 \mathrm{kHz}$. 


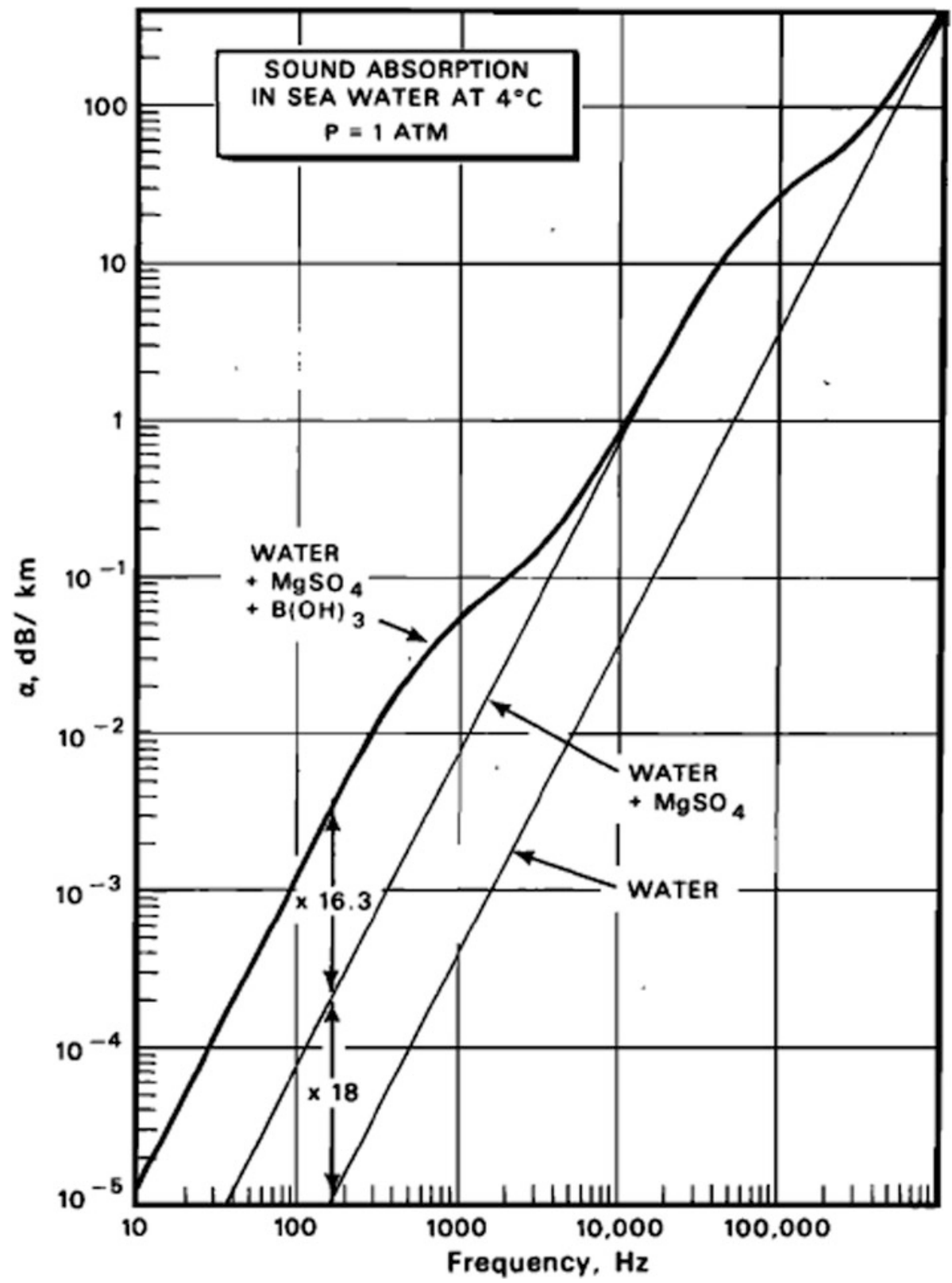

Fig. 14.7 Attenuation of sound in "standard" seawater [26] with salinity of $3.5 \%$ and $\mathrm{pH}=8.0$ at $4{ }^{\circ} \mathrm{C}$ [18]. Below $1 \mathrm{MHz}$, relaxation attenuation due to association-dissociation of boric acid, $\mathrm{B}(\mathrm{OH})_{3}$, and magnesium sulfate, $\mathrm{MgSO}_{4}$, dominates the "classical" contributions

The attenuation coefficient has the expected form, based on Eq. (14.40), and is plotted as a function of frequency in Fig. 14.7.

$$
\frac{\alpha}{f^{2}}=\frac{A_{\mathrm{B}(\mathrm{OH})_{3}} f_{\mathrm{B}(\mathrm{OH})_{3}}}{f^{2}+f_{\mathrm{B}(\mathrm{OH})_{3}}^{2}}+\frac{P_{\mathrm{MgSO}_{4}} A_{\mathrm{MgSO}_{4}} f_{\mathrm{MgSO}_{4}}}{f^{2}+f_{\mathrm{MgSO}_{4}}^{2}}+A_{o} P_{o}
$$


Approximate expressions for the coefficients representing the relaxation strengths, $A$, and pressure correction factors, $P$, are provided by Fisher and Simmons [18] with more accurate values provided by François and Garrison [24, 25].

\subsection{Transmission Loss}

The fact that the bulk attenuation of sound in fluids is quadratic in the frequency has important consequences for ultrasonics ( $f>20 \mathrm{kHz}$ ) and for long-range sound propagation. At the extremely low frequencies, infrasound in the Earth's atmosphere can propagate around the entire globe, and the sound of breaking waves generated by a storm on the Pacific coast of the United States has been detected by a low-frequency microphone at the Bureau of Standards in Washington, DC. An International Monitoring System with 60 infrasound monitoring stations has been deployed globally to detect violations of the Comprehensive Nuclear-Test-Ban Treaty [27].

\subsubsection{Short and Very Short Wavelengths}

As discussed in Sect. 12.8.1, the Rayleigh resolution criterion implies that the smallest feature that can be resolved in an ultrasonic image will be limited by the wavelength of the sound used to produce the image. Since many ultrasonic imaging systems are used in biomedical applications, we can assume a speed of sound in biological tissue that is approximately equal to the speed of sound in water, $c_{\mathrm{H}_{2} \mathrm{O}}=$ $1500 \mathrm{~m} / \mathrm{s}$ [28]. To resolve an object that is about a millimeter would then require sound at a frequency, $f=c / \lambda \cong 1.5 \mathrm{MHz}$. At that frequency, the attenuation of sound in liver tissue is over $2 \mathrm{~dB} / \mathrm{cm}$, so a roundtrip transmission loss to go to a depth of $10 \mathrm{~cm}$ is $40 \mathrm{~dB}$.

Because the speed of sound in liquids is typically 200,000 times slower than the speed of light, it is possible to achieve optical wavelength resolution of about $5000 \AA=0.5 \mu \mathrm{m}$ using sound at a frequency of $3 \mathrm{GHz}$. In addition, acoustical microscopy produces image "contrast" due to variation in the acoustic absorption of the specimens and scattering that arises from the acoustic impedance mismatch between the specimen and the surrounding material due density and compressibility differences (see Sects. 12.6.1 and 12.6.2). Such sources of contrast will reveal completely different information about a specimen than can be deduced due to changes in optical index of refraction or optical reflectance. In addition, sound can penetrate an optically opaque object, and staining is not required for contrast enhancement. ${ }^{7}$

As mentioned, acoustic microscopy is limited by the fact that attenuation is such a strong function of frequency. At $1.0 \mathrm{GHz}$, the attenuation of sound in water is $200 \mathrm{~dB} / \mathrm{mm}$ [29]. Despite the high attenuation loss, acoustic microscopes can image red blood cells acoustically at $1.1 \mathrm{GHz}$ with a resolution equivalent to an oil-immersion optical microscope at a magnification of 1000 [30]. Subcellular details as small as $0.1-0.2 \mu \mathrm{m}$ (e.g., nuclei, nucleoli, mitochondria, and actin cables) have been resolved due to the extraordinary contrast that can differentiate various cytoplasmic organelles [31].

The greatest resolution that has been achieved using acoustical microscopy has been accomplished in superfluid helium at temperatures near absolute zero, which has a sound speed, $c_{1} \cong 240 \mathrm{~m} / \mathrm{s}$, a speed that is even lower than the speed of sound in air. Since the dynamics of liquid helium at temperatures below $T_{\lambda}=2.17 \mathrm{~K}$ are determined by quantum mechanics, the attenuation mechanisms

\footnotetext{
${ }^{7}$ Encapsulated microbubbles are used occasionally to provide the ultrasonic image enhancement equivalent of "staining" in optical imaging.
} 
are different than those for classical fluids, which also gives it a much smaller attenuation. At low temperatures, $T<0.5 \mathrm{~K}$, the phonon mean free path is controlled by scattering from "rotons," which are quantized collective excitation of the superfluid [32]. Sound wavelengths in liquid helium shorter than $2000 \AA=0.2 \mu \mathrm{m}$, in a non-imaging experiment, at frequencies of $1.0 \mathrm{GHz}$, had been studied before 1970 by Imai and Rudnick [33].

\subsubsection{Very Long Wavelengths}

At the opposite extreme, at much lower frequencies, the absorption can be quite small. Although the worldwide network of infrasound monitoring sites, using electronic pressure sensors and sophisticated signal processing, that is being used to assure compliance with the Comprehensive Nuclear-Test-Ban Treaty has already been mentioned [27], the most famous measurement of long-distance infrasound propagation was made using barometers.

On 27 August 1883, the island of Krakatoa, in Indonesia east of Java, was destroyed by an immense volcanic explosion. The resulting pressure wave was recorded for days afterward at more than 50 weather stations worldwide. Several of those stations recorded as many as seven passages of the wave as it circled the globe:

"The barograph in Glasgow recorded seven passages: at 11 hours, 25 hours, 48 hours, 59 hours, 84 hours,

94 hours, and 121 hours (5 days) after the eruption." [34]

About $4 \mathrm{~h}$ after the explosion, the pressure pulse appeared on a barograph in Calcutta. In $6 \mathrm{~h}$, the pulse reached Tokyo; in $10 \mathrm{~h}$, Vienna; and in $15 \mathrm{~h}$, New York. The period of the pulse was between 100 and $200 \mathrm{~s}$ corresponding to a fundamental frequency of about $7 \mathrm{mHz}$. Its propagation velocity was between 300 and $325 \mathrm{~m} / \mathrm{s}$ [35]. Although that is close to the speed of sound in air near room temperature, the wave was similar to a shallow water gravity wave in which the height of the atmosphere rose and fell with the passage of the wave [36].

\subsection{Quantum Mechanical Manifestations in Classical Mechanics}

"The major role of microscopic theory is to derive phenomenological theory." G. E. Uhlenbeck ${ }^{8}$

Although acoustics is justifiably identified as a field of classical phenomenology, there are many acoustical effects that have their origin in the microscopic theory of atoms and therefore manifest macroscopic behaviors that can only be explained in terms of quantum mechanics. The effects of these "hidden variables" have been manifest throughout this textbook starting with the damping of simple harmonic oscillators that connects the "system" to the environment, thus producing Brownian motion [37], which was related to the more general theory coupling fluctuations and dissipation [38] in Chap. 2. ${ }^{9}$

This theme recurred in Chap. 7 when the quantization of energy levels for molecular vibration and rotation influenced the specific heat of gases and in Chap. 9 where a simple kinetic theory of gases was used to determine the pressure and temperature variation of viscosity and thermal conductivity. Now we see in this chapter how structural relaxations in water [23], like those in Fig. 5.23 for the four

\footnotetext{
${ }^{8}$ George Eugène Uhlenbeck (1900-1988) was a Dutch theoretical physicist who, with fellow Dutchman, Samuel Goudsmit (1902-1978), first proposed quantized "spin" as the internal degree of freedom for electrons.

${ }^{9}$ Lars Onsager (1903-1976) was the Norwegian-born physical chemist and theoretical physicist who received the Nobel Prize in chemistry, in 1968, for the reciprocal relations between fluctuations and dissipation that are now referred to as "Onsager reciprocity."
} 
crystalline structures of plutonium [39], scattering of phonons and rotons in superfluids [32], or molecular vibrations in $F_{2}$ [19] and collision times in gas mixtures [15], or chemical reactions $[24,25]$, manifest themselves in the attenuation of sound.

These internal relaxation effects have been incorporated into our phenomenological theory through the introduction of an additional dissipative process that has been quantified by the introduction of a frequency-dependent parameter, $\zeta$, that shares the same units with the coefficient of shear viscosity, $\mu$. That coincidence has led to this new parameter being called the coefficient of "bulk viscosity" (or sometimes "second viscosity"), even though it is independent of the shear deformation of the fluid and is not the source of momentum transport.

Talk Like an Acoustician

$\begin{array}{ll}\text { Viscoelasticity } & \text { Mean free path } \\ \text { Viscous drag } & \text { Kinetic theory } \\ \text { Thermal relaxation } & \text { Einstein summation convention } \\ \text { Thermoviscous boundary layer } & \text { Bulk viscosity } \\ \text { Spatial attenuation coefficient } & \text { Second viscosity } \\ \text { Temporal attenuation coefficient } & \text { Le Châtelier's principle } \\ \text { Shear strain } & \text { Nondimensional frequency } \\ \text { Hydrostatic strain } & \text { Vibrational relaxation time } \\ \text { Collision time } & \text { Structural relaxation } \\ \text { Enthalpy function } & \text { Association-dissociation reactions } \\ \text { Legendre transformation } & \text { Kramers-Kronig relations } \\ \text { Maxwell relations } & \\ \text { Relaxation time } & \end{array}$

\section{Exercises}

1. Bulk attenuation and reverberation time. An expression was provided in Eq. (13.29) to incorporate the attenuation in air contained within an enclosure into the expression for reverberation time. A "useful correlation" was provided in Eq. (13.30) that was applicable for $1500 \mathrm{~Hz} \leq f \leq 10,000 \mathrm{~Hz}$ and for relative humidity in the range $20 \% \leq R H \leq 70 \%$.

(a) Average frequency dependence. Table 14.1 (left) provides the attenuation in $\mathrm{dB} / \mathrm{km}$ from the ANSI/ASA standard for the frequencies within the range specified at $20{ }^{\circ} \mathrm{C}$ for $R H=50 \%$ [17]. Plot the $\log _{10}$ of the spatial attenuation, $\alpha$, in $\mathrm{m}^{-1}$ vs. the $\log _{10}$ of frequency, $f$, in $\mathrm{kHz}$, to determine the power law dependence on frequency (see Sect. 1.9.3). Is your result proportional to $f^{1.7}$ to within the statistical uncertainty of your least-squares fit? Keep in mind that attenuation expressed in $[\mathrm{dB} / \mathrm{m}]$ must be multiplied by $0.1151 \cong\left[10 \log _{10}\left(e^{2}\right)\right]^{-1}$ to convert to $\mathrm{m}^{-1}$ (sometimes including the dimensionless "Nepers" to report results in Nepers $/ \mathrm{m}$ ).

(b) Humidity dependence. Table 14.1 (right) also includes the attenuation in $\mathrm{dB} / \mathrm{km}$ at $4.0 \mathrm{kHz}$ and $20{ }^{\circ} \mathrm{C}$ for $20 \% \leq R H \leq 70 \%$. The "useful correlation" claims that the correction to frequency dependence for variations in relative humidity should be linear in $(50 \% / R H)$. How close is that presumed humidity dependence to values in the table for variation in relative humidity at $4.0 \mathrm{kHz}$ ?

2. The mother of all PA systems. Shown in Fig. 14.8 is a loudspeaker that can produce 30,000 watts of acoustic power by modulating a pressurized air stream (like a siren) using a cylindrical "valve" mounted on a voice coil, like that used for an electrodynamic loudspeaker. That sound source, located at the apex of the horn, is called a "modulated airstream loudspeaker." [40] 
Fig. 14.8 A very large horn loudspeaker mounted on an 18-wheel tractortrailer. (Photo courtesy of Wiley Labs)

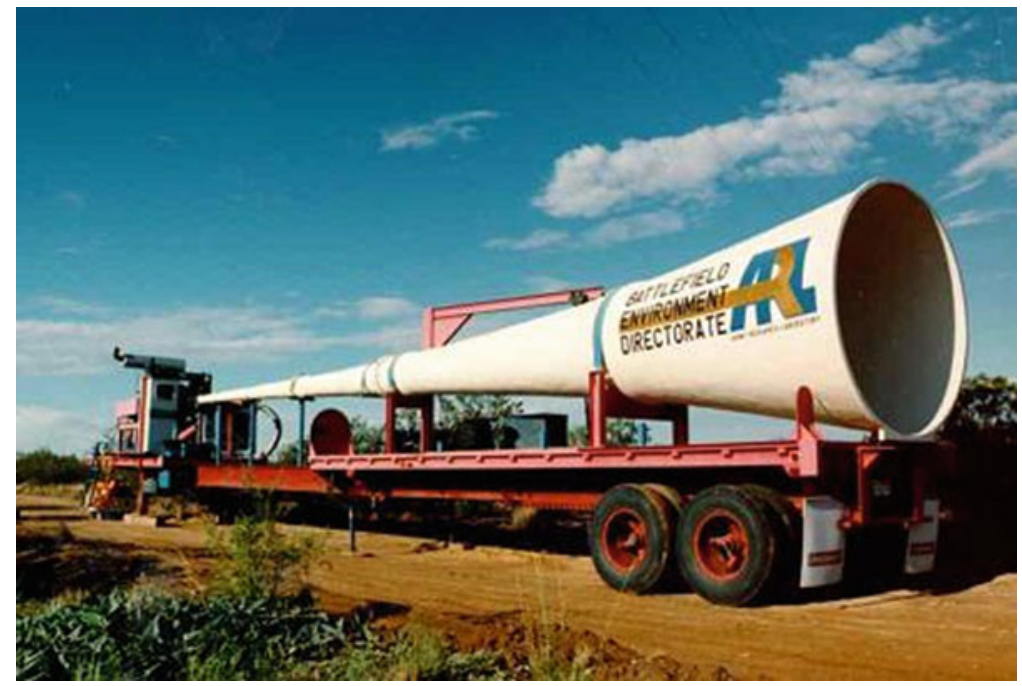

Such a public address system was developed to tell illiterate enemy combatants, in their native language, to put down their weapons and surrender from a distance that is greater than the distance that could be traversed by artillery shells. Although the bandwidth of telephone speech for very good intelligibility is generally $300 \mathrm{~Hz}$ to $3.4 \mathrm{kHz}$, for this problem, we will focus on the propagation of a $1 \mathrm{kHz}$ pure tone. Since this system was deployed in desert terrain, assume that $R H=10 \%$, $T_{m}=+50{ }^{\circ} \mathrm{C}=122^{\circ} \mathrm{F}$, and $p_{m}=100 \mathrm{kPa}$.

(a) Sound level at $100 \mathrm{~m}$. Assuming hemispherical spreading and no sound absorption, what is the root-mean-square acoustic pressure amplitude if the source produces $30 \mathrm{~kW}$ of acoustic power and any nonlinear effects that might cause harmonic distortion (see Sect. 15.2.3) can be neglected? Also neglect any refractive effects due to sound speed gradients caused by temperature or wind as discussed in Sect. 11.3. Report your results as both in r.m.s. pressure amplitude and in $\mathrm{dB}$ re: $20 \mu \mathrm{Pa}_{\mathrm{rms}}$.

(b) Greater distances. Repeat part $a$, but determine the sound pressure at $1.0 \mathrm{~km}$ and $3.0 \mathrm{~km}$, again neglecting attenuation.

(c) Include attenuation. Determine the spatial attenuation coefficient under these conditions at $1.0 \mathrm{kHz}$ and use it to reduce the sound pressure at $0.1 \mathrm{~km}, 1.0 \mathrm{~km}$, and $3.0 \mathrm{~km}$ below that obtained due only to hemispherical spreading.

3. Pump wave attenuation for a parametric array. The generation of highly directional sound beams from the nonlinear acoustical interaction of two colinear high-frequency sound beams will be discussed in Sect. 15.3.3. Calculate the exponential attenuation length, $\ell=\alpha^{-1}$, of a typical $40 \mathrm{kHz}$ beam in dry air, $R H=0 \%$, and moist air with $R H=60 \%$ using the graph in Fig. 14.5.

4. Siren. The siren shown in Fig. 14.9 consumed $2500 \mathrm{ft}^{3} / \mathrm{min}$ of air at a pressure of 5 psi above ambient to produce 50 horsepower of acoustic power at $500 \mathrm{~Hz}$, and did so with $72 \%$ efficiency [41].

(a) Hydraulic power. How much time-averaged power, $\left\langle\Pi_{h y}\right\rangle_{t}=(\Delta p)|U|$, is available, in watts and in horsepower, from the specified volume flow rate, $U$, and the available pressure drop, $\Delta p$ ?

(b) Hemispherical spreading. Assuming the mean temperature during the measurement was $T_{m}=20^{\circ} \mathrm{C}$ and $p_{m}=100 \mathrm{kPa}$, what would be the root-mean-square pressure a distance of 

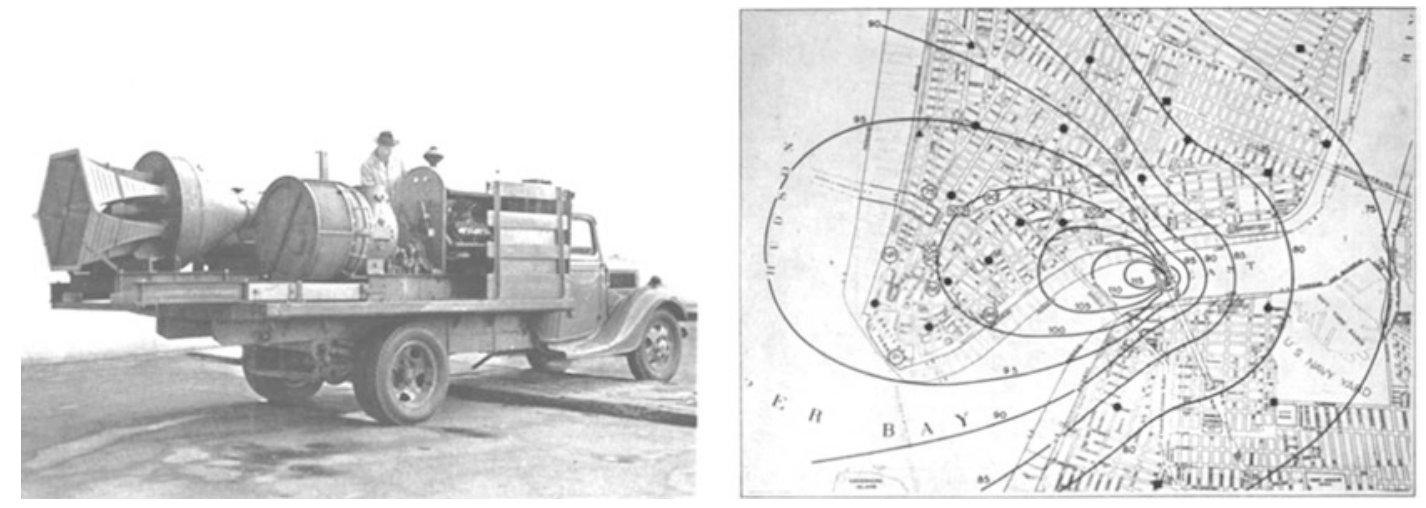

Fig. 14.9 (Left) Photograph of a 50-horsepower siren and compressor mounted on a truck. The intake filter and compressor are on the near side, and the exponential horn is farther away. (Right) Equal loudness contours measured throughout lower Manhattan when the siren was placed on the Manhattan Bridge facing toward the Financial District. The siren's directivity index was $11.8 \mathrm{~dB}$ at $500 \mathrm{~Hz}$ (see Sect. 12.8.2) [41]

$1000 \mathrm{ft}$. from the siren if it produced a sound power of 50 horsepower? Report your results both in pressure and $\mathrm{dB}$ re: $20 \mu \mathrm{Pa}_{\mathrm{rms}}$.

(c) Include attenuation. If $R H=50 \%$, how much additional loss, in $\mathrm{dB}$, would be produced by absorption at the same distance?

5. The SOFAR channel. One method for locating pilots who crash over the ocean is to drop an explosive sound source that is set to detonate at a depth that is equal to the axis of the deep sound channel that is created by a sound speed profile like the one shown in Fig. 11.8. In that figure, the axis of the sound channel is $1112 \mathrm{~m}$ below the ocean's surface. Sound that is trapped in that channel will spread cylindrically, rather than spherically, beyond a transition distance that will be assumed to be much shorter than the distance of interest, so the sound amplitude will decrease in proportion to $\sqrt{R}$, where $R$ is the distance between the source and the receiver.

(a) Cylindrical spreading. What would be the loss, in $\mathrm{dB}$, due to cylindrical spreading over $5000 \mathrm{~km}$ relative to the level $1 \mathrm{~km}$ from the source?

(b) Include attenuation. Using the results for seawater in Fig. 14.7, what would be the spatial attenuation, in $\mathrm{dB}$, that would have to multiply the cylindrical spreading loss over $5.0 \mathrm{~km}$ calculated in part (a) for sound with a frequency of $100 \mathrm{~Hz}$ ? Repeat for the loss due to attenuation in seawater after $5000 \mathrm{~km}$.

\section{High-frequency relaxational attenuation constant for air and water.}

(a) Limiting frequency dependence. Using Eq. (14.40), show that for frequencies well above the highest relaxational frequency, $f_{R}$, the attenuation of sound is independent of frequency.

(b) Relaxational attenuation constant (bulk viscosity) of air. In Eq. (14.42), the measured highfrequency limit of the spatial attenuation constant in air is $\alpha_{\text {air }} / f^{2}=1.84 \times 10^{-11} \mathrm{~s}^{2} / \mathrm{m}$. Calculate $\alpha_{\text {classical }} f^{2}$ for air at atmospheric pressure and $20{ }^{\circ} \mathrm{C}$, and use both high-frequency results (i.e., with and without relaxation effects) to determine the value for $\zeta_{\text {air }}$ in the highfrequency limit.

(c) Relaxational attenuation constant (bulk viscosity) of pure water. The measured highfrequency limit of the spatial attenuation constant in pure water at $4{ }^{\circ} \mathrm{C}$ is $\lim _{f \gg f_{R}} \alpha_{\mathrm{H}_{2} \mathrm{O}} / f^{2}=$ $2.5 \times 10^{-14} \mathrm{~s}^{2} / \mathrm{m}$. Based on $\alpha_{\text {classical }} / f^{2}$ for pure water at atmospheric pressure and $4{ }^{\circ} \mathrm{C}$, determine the value for $\zeta_{\mathrm{H}_{2} \mathrm{O}}$ in the high-frequency limit. 


\section{References}

1. M. Greenspan, Transmission of sound waves in gases at very low pressures, in Physical Acoustics, vol. 2A, (Academic Press, 1965), pp. 1-45

2. L.D. Landau, E.M. Lifshitz, Fluid Mechanics, 2nd edn (Butterworth-Heinemann, 1987). See §79; ISBN 0-75062767-0

3. H. Lamb, Hydrodynamics, 6th edn (Cambridge, 1932), reprinted (Dover, 1945). See §30; ISBN 0-486-60256-7

4. G.G. Stokes, On the theories of the internal friction of fluids in motion and the equilibrium and motion of elastic solids. Philos. Trans. (Cambridge) 8, 287-302 (1845)

5. R. B. Lindsay, Acoustics: Historical and Philosophical Development (Dowden, Hutchinson \& Ross, 1973). p. 261; ISBN 0-87933-015-5

6. M.J. Stefan, Über den Einfluss der inneren Reibung in der Luft auf die Schallbewegung. Sitzber. Akad. Wiss. Wein 53, 526-537 (1866)

7. G. Krichhoff, Über den Einfluss der Warmeleitung in einen Gase auf die Schallbewegung. Ann. Phys. Chem. 134, 177-193 (1868). English translation: On the influence of thermal conduction in a gas on sound propagation, in Physical Acoustics, ed. by R.B. Lindsay (Dowden, Hutchinson \& Ross, 1974). pp. 7-19

8. A. Bejan, Advanced Engineering Thermodynamics, 2nd edn (Wiley, 1997). See §4.4; ISBN 0-471-14880-6

9. F. Reif, Fundamentals of Statistical and Thermal Physics (McGraw-Hill, 1965), See $\$ 5.5$

10. American Institute of Physics Handbook, 3rd edn, ed. by D.W. Grey (McGraw-Hill, 1972). ISBN 07-001485-X

11. J.J. Markham, R.T. Beyer, R.B. Lindsay, Absorption of sound in fluids. Rev. Mod. Phys. 23(4), 353-411 (1951). See Eq. (A-13)

12. H.E. Bass, L.C. Sutherland, A.J. Zuckerwar, D.T. Blackstock, D.M. Hester, Erratum: Atmospheric absorption of sound: Further developments. J. Acoust. Soc. Am. 97, 680-683 (1995), J. Acoust. Soc. Am. 99(2), 1259 (1996)

13. J.T.R. Watson, R.S. Basu, J.V. Sengers, An improved representative equation for the dynamic viscosity of water substance. J. Phys. Chem. Ref. Prop. 9(4), 1255-1290 (1980)

14. J.M.M. Pinkerton, A pulse method for the measurement of ultrasonic absorption in liquid: Results for water. Nature 160, 128-129 (1947)

15. M. Greenspan, Rotational relaxation in nitrogen, oxygen, and air. J. Acoust. Soc. Am. 31(2), 155-160 (1959)

16. A.D. Pierce, Acoustics: An Introduction to its Physical Principles and Applications, 2nd edn (Acoustical Society of America, 1989). ISBN 0-88318-612-8, pp 554

17. R.J. Peppin, A. Scharine, Methods for Calculation of the Absorption of Sound in the Atmosphere, Am. Nat. Standard ANSI/ASA S1.26-2014. See \$5.2.1

18. F.H. Fisher, V.P. Simmons, Sound absorption in sea water. J. Acoust. Soc. Am. 62(3), 558-564 (1977)

19. F.D. Shields, Thermal relaxation in fluorine. J. Acoust. Soc. Am. 34(3), 271-274 (1962)

20. F.D. Shields, R.T. Lagemann, Tube corrections in the study of sound absorption. J. Acoust. Soc. Am. 29(4), 470-475 (1957)

21. H. E. Bass, L. C. Sutherland, A. J. Zuckerwar, D. T. Blackstock, and D. M. Hester, Atmospheric absorption of sound: Further developments. J. Acoust. Soc. Am. 97(1), 680-683 (1995), as corrected in the erratum, J. Acoust. Soc. Am. 99(2), 1259 (1996)

22. F.E. Fox, G.D. Rock, Compressional viscosity and sound absorption in water at different temperatures. Phys. Rev. 70 $(1 / 2), 68-73$ (1946)

23. L. Hall, The origin of ultrasonic absorption in water. Phys. Rev. 73(7), 775-781 (1948)

24. R.E. François, G.R. Garrison, Sound absorption based on ocean measurements. Part I: Pure water and magnesium sulfate contributions. J. Acoust. Soc. Am. 72(3), 896-907 (1982)

25. R.E. François, G.R. Garrison, Sound absorption based on ocean measurements: Part II - Boric acid contribution and equation for total absorption. J. Acoust. Soc. Am. 72(6), 1879-1890 (1982)

26. J. Lyman, R.H. Fleming, Composition of sea water. J. Mar. Res. 3, 134-146 (1940)

27. H.E. Bass, J. Bhattacharyya, M.A. Garcés, M. Hedlin, J.V. Olson, R.L. Woodward, Infrasound. Acoust. Today 2(1), 9-19 (2006)

28. J.C. Bamber, Speed of sound, in Principles of Medical Ultrasonics, 2nd edn, ed. by C.R. Hill, J.C. Bamber, G.R. ter Haar (Wiley, 2005). See Fig. 5.3, pg. 178; ISBN 0-470-09396-X

29. R.A. Lemons, C.F. Quate, Acoustic microscopy: Biomedical application. Science 181(4191), 905-911 (1975)

30. C.F. Quate, A. Atalar, H.K. Wickramasinghe, Acoustic microscopy with mechanical scanning - A review. Proc. IEEE 67(8), 1092-1114 (1975)

31. R.A. Johnston, A. Atalar, J. Heiserman, V. Jipson, C.F. Quate, Acoustic microscopy: Resolution of subcellular detail. Proc. Natl. Acad. Sci. U.S.A. 76(7), 3325-3329 (1979)

32. I.M. Khalatnikov, D.M. Chernikova, Zh. Eksperim. i Teor. Fiz. 49, 1957 (1965), 50, 411 (1966). Translation: Sov. Phys.-JETP 22, 1336 (1966), 23, 274 (1966)

33. J.S. Imai, I. Rudnick, Ultrasonic attenuation in liquid helium at 1 GHz. Phys. Rev. Lett. 22(14), 694-697 (1969) 
34. G.J. Symons (ed.), The Eruption of Krakatoa and Subsequent Phenomena. Report of the Krakatoa Committee of the Royal Society (Trübner \& Co., London, 1888)

35. T.B. Gabrielson, Krakatoa and the Royal Society: The Krakatoa explosion of 1883 . Acoust. Today 6(2), 14-18 (2010)

36. G.I. Taylor, Waves and tides in the atmosphere. Proc. R. Soc. A126(800), 169-183 (1929)

37. G.E. Uhlenbeck, S. Goudsmit, A problem in Brownian motion. Phys. Rev. 34, 145-151 (1929)

38. C.W. McCombie, Fluctuation theory in physical measurements. Rep. Prog. Phys. 16, 266-319 (1953)

39. Y. Suzuki, V.R. Fanelli, J.B. Betts, F.J. Freibert, C.H. Mielke, J.N. Mitchell, M. Ramos, T.A. Saleh, A. Migliori, Temperature dependence of elastic moduli of polycrystalline $\beta$ plutonium. Phys. Rev. B 84, 064105 (2011)

40. V. Salmon, J.C. Burgess, Modulated airstream loudspeaker. J. Acoust. Soc. Am. 29, 767 (1957)

41. R.C. Jones, A fifty horsepower siren. J. Acoust. Soc. Am. 18(2), 371-387 (1946)

Open Access This chapter is licensed under the terms of the Creative Commons Attribution 4.0 International License (http://creativecommons.org/licenses/by/4.0/), which permits use, sharing, adaptation, distribution and reproduction in any medium or format, as long as you give appropriate credit to the original author(s) and the source, provide a link to the Creative Commons license and indicate if changes were made.

The images or other third party material in this chapter are included in the chapter's Creative Commons license, unless indicated otherwise in a credit line to the material. If material is not included in the chapter's Creative Commons license and your intended use is not permitted by statutory regulation or exceeds the permitted use, you will need to obtain permission directly from the copyright holder. 